\title{
wiiw
}

\section{NOVEMBER 2016}

\section{Working Paper 132}

\section{Import Demand Elasticities Revisited}

Mahdi Ghodsi, Julia Grübler, Robert Stehrer

The Vienna Institute for International Economic Studies Wiener Institut für Internationale Wirtschaftsvergleiche 



\title{
Import Demand Elasticities Revisited
}

\author{
MAHDI GHODSI \\ JULIA GRÜBLER \\ ROBERT STEHRER
}

Mahdi Ghodsi and Julia Grübler are Research Economists at the Vienna Institute for International Economic Studies (wiiw). Robert Stehrer is Scientific Director of wiiw.

This paper was produced as part of the PRONTO (Productivity, Non-Tariff Measures and Openness) project funded by the European Commission under the 7th Framework Programme, grant agreement no: 613504 . 



\section{Abstract}

In this paper, we present import demand elasticities estimated for 167 countries over 5,124 products at the six-digit level of the Harmonised System. Following the semiflexible translog GDP function approach proposed by Kee et al. (2008), we estimate unilateral import demand elasticities for the period 1996-2014. Results are differentiated by country and product characteristics. South Asia and North America are associated with the most elastic import demand. Countries exhibiting the highest average elasticities belong to the economically most important countries in their respective regions, while countries with the lowest import demand elasticities are typically small island states. Import-weighted results suggest that especially countries rich in natural resources - particularly fossil fuels - are facing an inelastic import demand, with the agrifood sector for these states being more price-responsive than the manufacturing sector. Demand is found to be least price-sensitive for machinery and electrical equipment, and most price-elastic for the energy sectors. Distinguishing between the use of products, the highest import demand elasticities are associated with intermediate goods, which appears particularly noteworthy in the context of an increasing importance of global value chains, the global trade slowdown since 2011 and ongoing negotiations of mega-regional trade deals.

Keywords: international trade, import demand, elasticity

JEL classification: D12, F14 



\section{CONTENTS}

1. Introduction ........................................................................................................................................

2. The theoretical framework ..................................................................................................................... 2

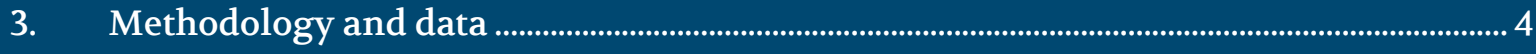

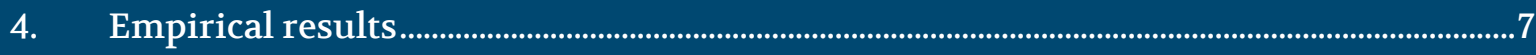

5. Robustness .............................................................................................................................................. 22

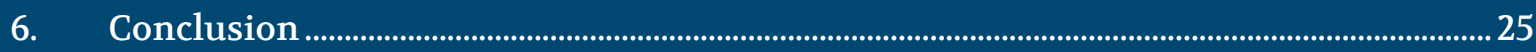

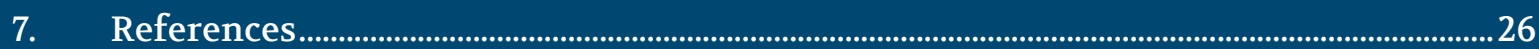

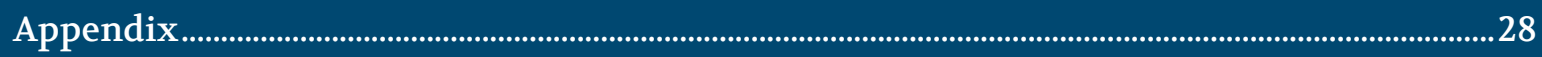




\section{TABLES AND FIGURES}

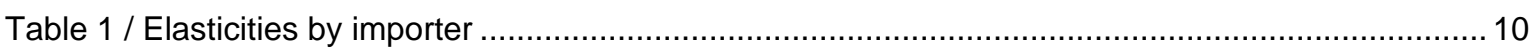

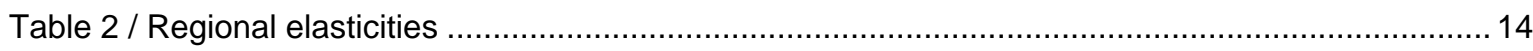

Table 3 / Regression of binding import demand elasticities on country characteristics ...................... 16

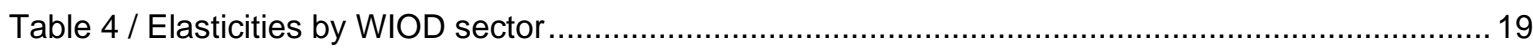

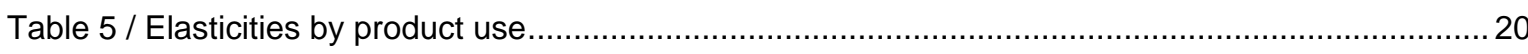

Table 6 / Regression of binding import demand elasticities on product characteristics .......................21

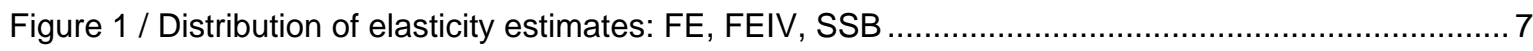

Figure 2 / Distribution of elasticity estimates at the HS 6 -digit level........................................... 8

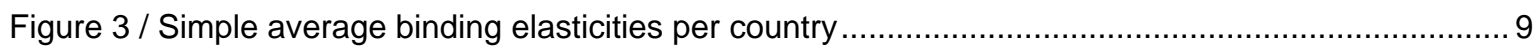

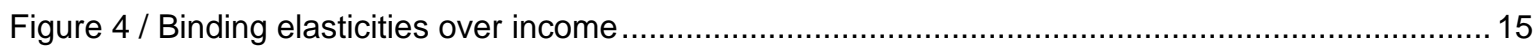

Figure 5 / Binding simple average elasticities per HS Section ..................................................... 18

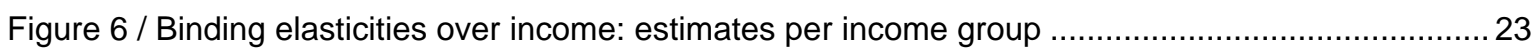

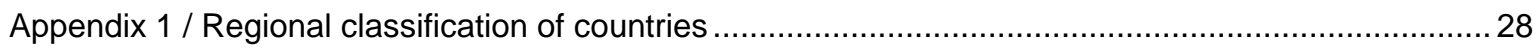

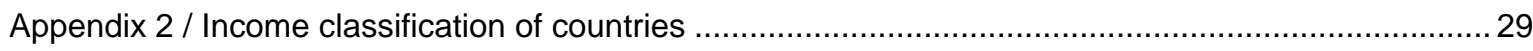




\section{Introduction}

The set of applied trade policy instruments is constantly increasing. In addition to traditional trade policy tools such as tariffs or quotas, non-tariff measures such as sanitary and phytosanitary measures, technical barriers to trade or antidumping practices feature prominently in ongoing trade negotiations. The number of trade agreements as well as their geographical scope and depth of their agendas is surging. Mega-regional trade agreements such as the Transatlantic Trade and Investment Partnership (TTIP) between the EU and the United States, the Transpacific Partnership (TPP) centred around the US, and the Regional Comprehensive Economic Partnership (RCEP) including China bear the potential of exerting a substantial impact on quantities and prices of imported products.

In order to compare the impact of different trade policies it is often necessary to make use of import demand elasticities (e.g. Kee et al., 2009; Nizovtsev and Skiba, 2016) answering the question: What would be the percentage change in import quantities if the price of the imported good increased by $1 \%$ ?

Trade policy is frequently operational at the tariff line level. However, there are only few studies which allow the evaluation of demand elasticities for a broad set of products at the very disaggregated product level (e.g. Kee et al., 2008; Feenstra and Romalis, 2014). Available studies have a strong focus on either selected products (e.g. Panagariya et al., 2001; Altinay, 2007) and/or particular importers (e.g. Broda and Weinstein, 2006; Soderbery, 2015).

To the best of our knowledge, the investigation by Kee et al. (2008) is the only work that evaluated price elasticities of import demand for a wide range of products and countries, having the inherent additional advantage of rendering elasticities across countries and products more comparable through the application of a single methodology and dataset for all.

Overall, Kee et al. (2008) estimated more than 300,000 import demand elasticities across 117 countries for about 4,900 products at the 6-digit level of the Harmonised System (HS revision 1988) for the period 1988-2001. Their estimates are frequently used in various policy analysis (e.g. Kee et al., 2009; Maoz, 2009; Bratt, 2014; Peterson and Thies, 2014; Beghin et al., 2015).

This paper constitutes an update of their work by computing importer-specific import demand elasticities for the more recent period 1996-2014 (HS revision 1996) and presents differences across countries, regions and income levels, as well as by products and sectors. Improved data availability and the inclusion of products not considered in HS revision 1988 allows us to estimate about twice as many import demand elasticities for 167 importing countries and 5124 products.

The remainder of this paper is structured as follows. Section 2 explains the theoretical framework. Section 3 describes the data used and the empirical strategy. Section 4 presents the empirical results and Section 5 discusses the robustness of the findings. The final section concludes. 


\section{The theoretical framework}

In this section, we briefly outline the approach suggested by Kee et al. (2008) to compute importerspecific import demand elasticities - i.e. the change in the total import quantity of a specific product (in \%) due to an increase of its price (by 1\%).

The starting point for Kee et al. (2008) is based on Kohli's (1991) GDP function approach. In an economy with $N$ products and $M$ factors of production, the optimal net output vector $q^{t}$ of an economy (i.e. output including exports and reduced by imports) maximises the value of goods produced in the economy $G^{t}\left(\tilde{p}^{t} A^{t}, v^{t}\right)$ given exogenous world prices $\tilde{p}^{t}$, productivity $A^{t}$ and factor endowments $v^{t}$ :

$$
G^{t}\left(p^{t}, v^{t}\right) \equiv \max _{q^{t}}\left\{p^{t} q^{t}:\left(p^{t}, v^{t}\right)\right\}
$$

where $p^{t}$ is the productivity inclusive and thus country-specific price vector $\left(p^{t} \equiv \tilde{p}^{t} A^{t}\right)$. Positive numbers for $q^{t}$ refer to output for domestic demand or exports, while negative numbers refer to imported goods.

If good $n$ is an imported good then the derivative of the GDP function with respect to its price gives the GDP-maximising import demand function of good $n$ which does neither depend on income nor on utility.

$$
\frac{\partial G^{t}\left(p^{t}, v^{t}\right)}{\partial p_{n}^{t}}=q_{n}^{t}\left(p^{t}, v^{t}\right), \forall n=1, \ldots, N .
$$

In order to evaluate the GDP function empirically, Kee et al. (2008) employ a flexible translog GDP function with indices $n$ and $k$ indicating goods and $m$ and $l$ representing factors of production:

$$
\begin{aligned}
\ln G^{t}\left(p^{t}, v^{t}\right)= & a_{00}^{t}+\sum_{n=1}^{N} a_{n n}^{t} \ln p_{n}^{t}+\frac{1}{2} \sum_{n=1}^{N} \sum_{k=1}^{N} a_{n k}^{t} \ln p_{n}^{t} \ln p_{k}^{t}+\sum_{m=1}^{M} b_{m m}^{t} \ln v_{n}^{t} \\
& +\frac{1}{2} \sum_{m=1}^{M} \sum_{l=1}^{M} b_{m l}^{t} \ln v_{m}^{t} \ln v_{l}^{t}+\sum_{n=1}^{N} \sum_{m=1}^{M} c_{n m}^{t} \ln p_{n}^{t} \ln v_{m}^{t}
\end{aligned}
$$

The derivative of $\ln G^{t}\left(p^{t}, v^{t}\right)$ with respect to $\ln p_{n}^{t}$ gives the equilibrium share of good $n$ in GDP at period $t$ :

$$
\frac{\partial \ln G^{t}}{\partial \ln p_{n}^{t}}=\frac{1}{G_{n}^{t}\left(p^{t}, v^{t}\right)} q_{n}^{t}\left(p^{t}, v^{t}\right) p_{n}^{t} \equiv s_{n}^{t}\left(p^{t}, v^{t}\right)
$$

which, after imposing restrictions on the functional form of the translog GDP function to ensure that it is homogeneous of degree one with respect to prices and factor endowments and satisfies the symmetry property, results in: 


$$
s_{n}^{t}\left(p^{t}, v^{t}\right)=a_{0 n}^{t}+a_{n n}^{t} \ln p_{n}^{t}+\sum_{k \neq n}^{N} a_{n k}^{t} \ln p_{k}^{t}+\sum_{m=1}^{M} c_{n m}^{t} \ln v_{m}^{t}, \quad \forall n=1, \ldots, N
$$

$s_{n}^{t}$ is the share of good $n$ in GDP (with negative values assigned to imports, and positive values associated with output and exports). Under consideration of the translog parameters of the GDP function, the derivative of $s_{n}^{t}$ with respect to prices $p_{n}^{t}$ is given as

$$
\frac{\partial s_{n}^{t}}{\partial p_{n}^{t}}=\underbrace{\frac{q_{n}^{t}}{G^{t}}+p_{n}^{t} \frac{\frac{\partial q_{n}^{t}}{\partial p_{n}^{t}}}{G^{t}}-\frac{q_{n}^{t} p_{n}^{t}}{\left(G^{t}\right)^{2}} \frac{\partial G^{t}}{\partial p_{n}^{t}}}_{\text {see eq.(4) }}=\underbrace{a_{n n}^{t} \frac{1}{p_{n}^{t}}}_{\text {see eq. (5) }}
$$

where $a_{n n}^{t}$ is a translog parameter stemming from the translog GDP function that captures the change in the share of good $n$ in GDP (which by construction is negative for imported products) when the price of good $n$ increases by $1 \%$. The multiplication of both sides by $p_{n}^{t}$ and rearranging terms ${ }^{1}$ gives the result for the import demand elasticity of imported good $n$ :

$$
\varepsilon_{n n}^{t} \equiv \frac{\partial q_{n}^{t}\left(p^{t}, v^{t}\right)}{\partial p_{n}^{t}} \frac{p_{n}^{t}}{q_{n}^{t}}=\frac{a_{n n}^{t}}{s_{n}^{t}}+s_{n}^{t}-1 \leq 0, \forall s_{n}^{t}<0
$$

If the share of imports in GDP does not change due to changes in import prices $\left(a_{n n}^{t}=0\right)$, then the implied import demand is unitary elastic, meaning that an increase of the price $p_{n}^{t}$ by $1 \%$ induces a proportional decrease in quantities $q_{n}^{t}$ such that the share in GDP $s_{n}^{t}$ remains constant.

If $a_{n n}^{t}>0$, the share of the imported good $n$ in GDP decreases (i.e. $s_{n}^{t}$ becomes less negative), implying that demand is elastic, such that an increase in the price reduces quantities more than proportional. Finally, if $a_{n n}^{t}<0$, the share of imported good $n$ in GDP increases (i.e. $s_{n}^{t}$ becomes more negative) import demand must be relatively inelastic $\left(-1<\varepsilon_{n n}^{t}<0\right)$, as quantities respond less than proportionately to a change in prices. Thus, for small shares and goods in accordance with the law of demand it holds:

$$
\varepsilon_{n n}^{t}\left\{\begin{aligned}
{[-100 ;-1) } & \text { if } a_{n n}^{t}>0 \\
-1 & \text { if } a_{n n}^{t}=0 \\
(-1 ; 0] & \text { if } a_{n n}^{t}<0
\end{aligned}\right.
$$

1 The multiplication of both sides of equation (6) with $p_{n}^{t}$ and remembering that, (i) $\frac{\partial G^{t}}{\partial p_{n}^{t}}=q_{n}^{t}$, (ii) $\partial s_{n}^{t} \equiv q_{n}^{t} p_{n}^{t} / G^{t}$ and (iii) $\varepsilon_{n n}^{t} \equiv \frac{\partial q_{n}^{t}\left(p^{t}, v^{t}\right)}{\partial p_{n}^{t}} \frac{p_{n}^{t}}{q_{n}^{t}}$ results in $s_{n}^{t}+s_{n}^{t} \varepsilon_{n n}^{t}-\left(s_{n}^{t}\right)^{2}=a_{n n}^{t}$. 


\section{Methodology and data}

Empirically, Kee et al. (2008) implemented this strategy by using a parameterisation from a fully flexible to a semi-flexible translog function following Diewert and Wales (1988) and by restricting all translog parameters to be time invariant in order to handle the large number of goods at the HS 6-digit level. ${ }^{2}$ The resulting share equation is

$$
s_{n}^{t}\left(p^{t}, v^{t}\right)=a_{0 n}+a_{n n} \ln \frac{p_{n}^{t}}{\overline{p_{k}^{t}}}+\sum_{m=1, m \neq l}^{M} c_{n m} \ln \frac{v_{m}^{t}}{v_{l}^{t}}, \forall n=1, \ldots, N .
$$

where $p_{n}^{t}$ is measured using unit values of imports, $\overline{p_{k}^{t}}$ is a weighted average of the log prices of all non$n$ goods. Therefore, the share of good $n$ in GDP is a linear function of factor endowments and the price of good $n$ relative to an average price of all non- $n$ goods. Factors of production used in this analysis comprise labour, capital and agricultural land. Following Caves et al. (1982), Kee et al. approximate $\overline{\ln p_{k}^{t}}$ with the observed Tornqvist price index $\ln p_{-n}^{t}$ of all non- $n$ goods using the GDP deflator $p^{t}$.

$$
\ln p_{-n}^{t}=\frac{\left(\ln p^{t}-\bar{s}_{n}^{t} \ln p_{n}^{t}\right)}{\left(1-\bar{s}_{n}^{t}\right)} \text {, with } \bar{s}_{n}^{t}=\frac{\left(\bar{s}_{n}^{t}+\bar{s}_{n}^{t-1}\right)}{2}
$$

Pooling data across countries and years for each good $n$, while employing country and year fixed effects, the final share equation estimated by Kee et al. (2008) for each good $n$ takes the following form:

$$
s_{n i}^{t}\left(p_{n i}^{t}, p_{-n i}^{t}, v_{i}^{t}\right)=a_{0 n}+a_{n i}+a_{n}^{t}+a_{n n} \ln \frac{p_{n i}^{t}}{p_{-n i}^{t}}+\sum_{m=1, m \neq l}^{M} c_{n m} \ln \frac{v_{m i}^{t}}{v_{l i}^{t}}+u_{n i}^{t}, \quad \forall n=1, \ldots, N .
$$

where $a_{n i}$ and $a_{n}^{t}$ denote country and time fixed effects, respectively. It is assumed that the structural parameters of the semiflexible translog GDP function are common across countries up to a constant. Equation 11 can be estimated with data on importer-specific product shares in GDP, the GDP deflator, unit values, and information on factor endowments.

Final modifications allow (i) for the correction of a possible endogeneity bias by using instruments for unit values, and (ii) for the correction of a selection bias by following a two-step procedure.

The basic intuition of the import demand elasticity is that if prices increase, demand for these goods decreases. However, if an economy experiences a positive demand shock, prices might react to demand and increase, resulting in reversed causality and simultaneity bias. We therefore instrument the unit values of good $n$ by two measures:

First, we use the simple average of the Tornqvist price index for product $n$ computed over all countries except importing country $i$, i.e. over the rest of the world. The reasoning is that we expect world price indices of good $n$ to be positively correlated with the importing country's price index for the same product

2 The parameterisation from a fully flexible to a semi-flexible translog function reduces the number of parameters to be estimated from $\mathrm{N}(\mathrm{N}-1) / 2+\mathrm{N}$ to $\mathrm{N}$ diagonal elements of the substitution matrix. 
thereby affecting import demand. However, while a domestic demand shock might impact an economy's domestic and import prices, we do not expect that domestic demand for imported products is shaping price indices of the rest of the world.

Remembering from equation (10) that the price of non- $n$ goods can be expressed as the GDP deflator adjusted for the share and price of good $n$, the price index for good $n$ over all non- $i$ importing countries (indexed $j$ ) can be computed in a similar fashion:

$$
I V_{1}\left(\ln \frac{p_{n i}^{t}}{p_{-n i}^{t}}\right)=\ln \frac{\bar{p}_{n j}^{t}}{\bar{p}_{-n j}^{t}}=\underbrace{\ln \left(\sum_{j} \frac{p_{n j}^{t}}{J}\right)}_{\ln \left(\bar{p}_{n j}^{t}\right)}-\underbrace{\left(\frac{\ln \sum_{j} \frac{p_{j}^{t}}{J}-\sum_{j} \frac{\bar{s}_{n j}^{t}}{J} \ln \sum_{j} \frac{p_{n j}^{t}}{J}}{\left(1-\sum_{j} \frac{\bar{s}_{n j}^{t}}{J}\right)}\right)}_{\ln \left(\bar{p}_{-n j}^{t}\right)}, j \neq i
$$

A second instrument is the trade-weighted average distance of the importing country to its trading partners. The intuition being that the price of imported products is expected to be higher for products that have to be transported over greater distances, while distance might not be correlated with domestic demand for good $n$.

$$
I V_{2}\left(\ln \frac{p_{n i}^{t}}{p_{-n i}^{t}}\right)=\sum_{r} x_{r}^{t} \text { distance }_{r i}
$$

where distance $e_{r i}$ is the physical distance between importer $i$ and exporter $r$ and $x_{r}^{t}$ is the share of an exporter $r$ in total exports of good $n$ in period $t$.

Results using these instruments might, however, still suffer from a selection bias, as unit values entering our analysis are calculated based on positive import flows. Country and year fixed effects can reduce the bias resulting from unobserved variables. Yet, due to the possibility that zero trade flows in our data are the result of countries' selection not to import, we follow an amended form of the Heckman two-stage estimation procedure. In the first step of the two-stage estimation procedure, the selection equation (14a) evaluates the probability of non-zero trade flows. The dependent variable is equal to 1 if the share of good $n$ in country $i$ 's GDP is smaller than zero (i.e. imports are greater than zero). It is regressed on a product-specific term $\gamma_{0 n}$, time fixed effects $\gamma_{n}^{t}$, country fixed effects $\gamma_{n i}$, as well as the previously introduced instruments and factor endowments, captured in $z_{n i}^{t} . \epsilon_{i n t}$ is an error term.

From this first step, the inverse Mills ratio $\left(\phi_{n i}^{t}\right)$ is obtained, which enters the outcome equation (15) in the second step as an explanatory variable, which should solve the omitted variable bias in the presence of sample selection. A drawback of this procedure is, that probit model estimations with country fixed effects suffer from the incidental parameters problem. It means that as we are using a big panel data set incorporating many fixed effects, probit models are more likely to render biased and inconsistent estimates, as they do not converge to their true value as the number of parameters (i.e. fixed effects) increases with sample size. In line with Kee et al. (2008) we therefore substitute country fixed effects with time averages of the exogenous variables and instruments $\bar{z}_{n i}$ in the first stage (equation 14b).

$$
\begin{aligned}
& \operatorname{Prob}\left[s_{n i}^{t}<0\right]=\gamma_{0 n}+\gamma_{n}^{t}+\gamma_{n i}+\delta_{1 n} z_{n i}^{t}+\epsilon_{i h t}, \quad \forall n=1, \ldots, N . \\
& \operatorname{Prob}\left[s_{n i}^{t}<0\right]=\gamma_{0 n}+\gamma_{n}^{t}+\delta_{1 n} z_{n i}^{t}+\delta_{2 n} \bar{z}_{n i}+\epsilon_{i h t}, \quad \forall n=1, \ldots, N .
\end{aligned}
$$




$$
\begin{gathered}
\left(s_{n i}^{t} \mid s_{n i}^{t}<0\right)=a_{0 n}+a_{n}^{t}+a_{n n} \ln \frac{p_{n i}^{t}}{p_{-n i}^{t}}+\sum_{m=1, m \neq l}^{M} c_{n m} \ln \frac{v_{m i}^{t}}{v_{l i}^{t}}+d_{n} \bar{z}_{n i}+h_{n} \hat{\phi}_{n i}^{t}+u_{n i}^{t}, \\
\forall n=1, \ldots, N .
\end{gathered}
$$

Finally, using the average import shares of each importing country $i$ and estimates of $a_{n n}$ the resulting import demand elasticity of country $i$ for good $n$ is computed as

$$
\hat{\varepsilon}_{n n i} \equiv \frac{\partial q_{n}^{t}\left(p^{t}, v^{t}\right)}{\partial p_{n}^{t}} \frac{p_{n}^{t}}{q_{n}^{t}}=\frac{\widehat{a_{n n}}}{\overline{s_{n l}}}+\overline{s_{n l}}-1
$$

The data necessary for estimation was compiled from different sources. Import values and quantities were taken from the Commodity Trade Statistics Database (COMTRADE) for the period 1995-2014. It covers 5,221 products at the HS 6-digit (rev. 1996) level. Data on agricultural land in square kilometres was retrieved from the World Development Indicators (WDI) database of the World Bank and complemented by data provided by the Food and Agriculture Organization of the United Nations (FAO). Data on GDP, physical capital and labour was collected from the Penn World Tables 9.0 (Feenstra et al., 2015). 


\section{Empirical results}

On average, each HS 6-digit product in our sample was imported by 155 countries, with a minimum of 17 importers for petroleum oil obtained from bituminous minerals (HS 271094) and a maximum of 167 importers for 378 different products at the HS 6-digit level. Countries in the sample imported on average 4,790 products, ranging from a minimum of 1,593 products for Djibouti to 5,121 products for France. We dropped observations for which bilateral import values were reported but bilateral quantities were missing in order to avoid a bias of unit values entering our estimation procedure.

Following the methodology presented in Section 3, we performed three estimations: first, employing simple fixed effects (FE), second, introducing instrument variables to the fixed effects estimation procedure (FEIV) and finally, substituting the fixed effects approach by a two-step procedure to account for a possible sample selection bias (SSB).

Based on these results we constructed our final set of elasticity estimates. We based our decision when to replace FE results by FEIV results upon two criteria: (i) The Hansen J-statistic reports the validity of instruments, with the null hypothesis that instruments are exogenous. (ii) The Anderson-Rubin F-statistic shows whether instruments have an impact on the endogenous variable, with the null hypothesis that the endogenous regressors in the structural equation are jointly equal to zero. We therefore replaced FE estimates by FEIV results only if the Hansen J-statistic was greater than 0.1 and the Anderson-Rubin F-statistic was smaller than 0.1.

In addition to these two instrument variable criteria, when the coefficient of the inverse mills ratio $\left(h_{n}\right)$ in equation (15), indicating whether our results might suffer from sample selection bias, was found to be statistically significantly different form zero at the $10 \%$ level FEIV results were replaced by SSB results.

Figure 1 / Distribution of elasticity estimates: FE, FEIV, SSB
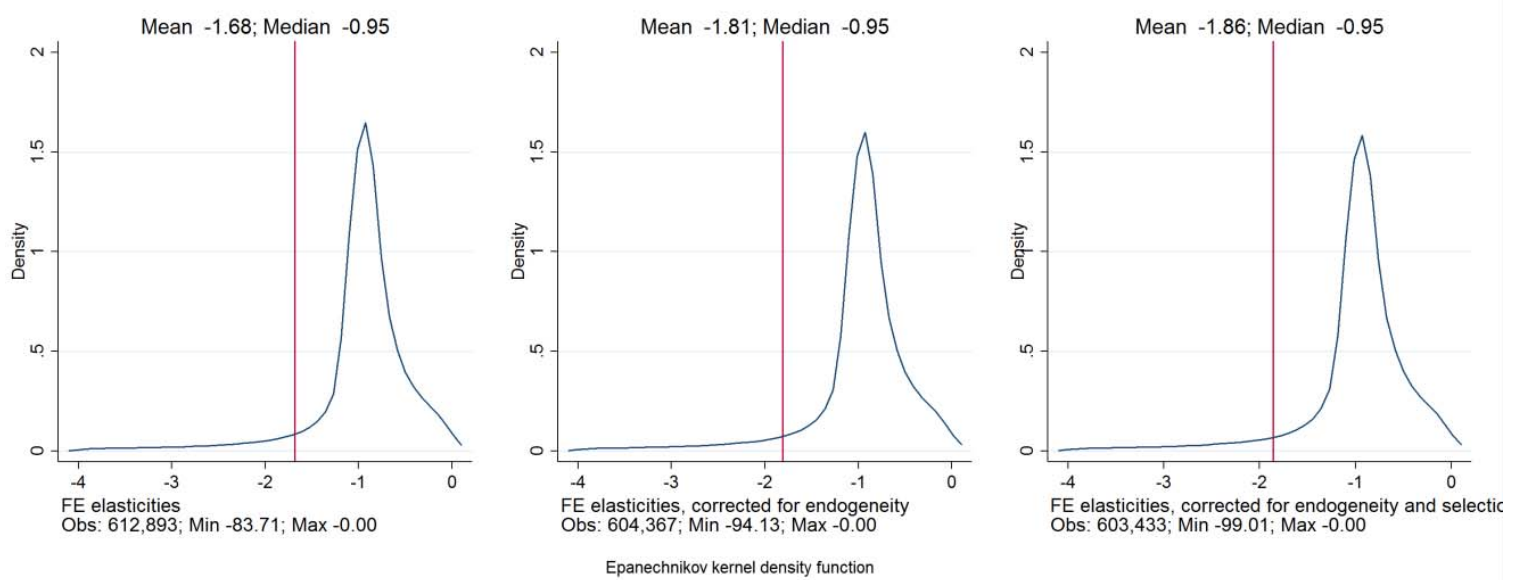
Figure 1 shows the distribution of elasticities along our modifications. Throughout, it looks quite similar, with mean elasticities smaller, i.e. more negative, than -1.6 but median elasticities larger than -1 . Corrections for endogeneity and a selection bias leave median values unchanged but shift mean values towards -2 .

For our preferred specification we additionally dropped observations where import values of one importer for one specific product never exceeded 10,000 USD per year during the period 1995-2014², which does not alter results on the median elasticity, but drastically reduces the highest elasticities from close to -100 to -25 .

Extreme values and potential outliers were dealt with in two steps: First, we dropped the tails $(0.5 \%$ from either side) of the distribution. Second, we dropped positive elasticities as we are not concerned with products that violate the law of demand, such as Giffen goods. These steps reduce the number observations from 687,927 to 548,625 import demand elasticity estimates, of which roughly $80 \%$ show to be significantly different from zero at the $10 \%$ level. We will henceforth refer to the latter as binding elasticities. Figure 2 shows the distribution of the elasticity estimates, with the left panel depicting all elasticities and the right panel presenting only binding elasticities.

\section{Figure 2 I Distribution of elasticity estimates at the HS 6-digit level}
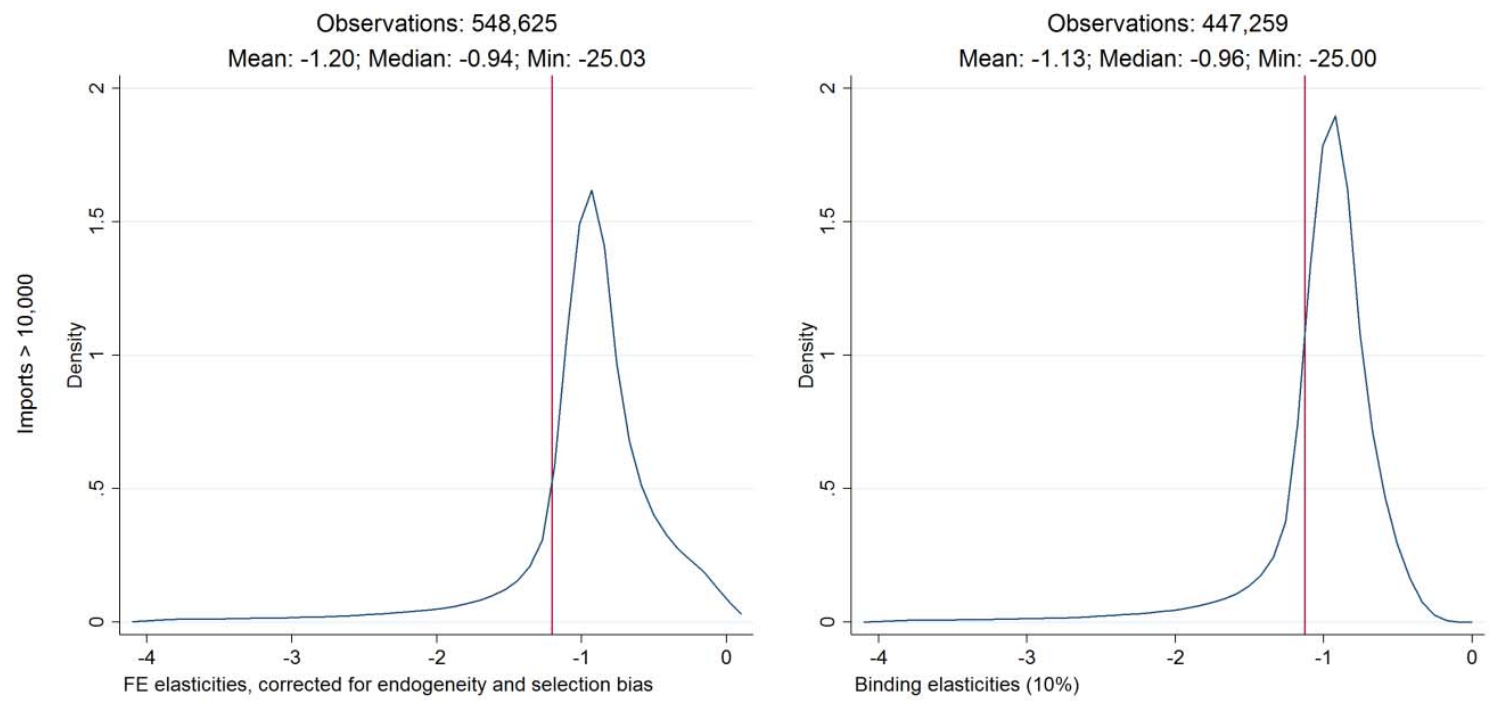

Note: Binding elasticities refer to estimates significantly different from zero at the $10 \%$ level.

While the distribution of our results on first sight very much resembles the findings of Kee et al. (2008) with a big spike around unitary elasticities and a quick flattening out of the distribution, our average elasticity of -1.20 is much less elastic than the mean elasticity reported by Kee et al. (2008) of -3.12 . As illustrated in Figure 2, our results suggest that the most elastic HS 6-digit product is facing an elasticity of -25.03 . However, the data provided by Kee et al. (2008) reaches from zero to -372.25 with 91 products attributable to 45 importing countries showing elasticities equal or greater than -300 .

3 Section 5 on the robustness of our findings also discusses result, when no import threshold is imposed. 


\subsection{ELASTICITIES BY IMPORTER}

While Figure 1 and Figure 2 have shown the distribution of our estimated import demand elasticities over all HS 6-digit products and importers, this section aims to discuss geographical patterns of this distribution. We start by discussing elasticity aggregates by country and proceed by computing regional average elasticities and finally illustrate average elasticities by income group.

Table 1 summarises our results per country. The first two columns report our findings as simple average (s.a.) elasticities. Results shown in the second and subsequent columns are restricted to products for which elasticities were found to be binding, i.e. significantly different from zero at the $10 \%$ level. The third column of Table 1 shows results of binding elasticities when import-weights are applied. Columns four and five split up these import-weighted results into the agri-food and the manufacturing sector.

Figure 3 illustrates simple average binding elasticities (corresponding to column two of Table 1) with a world map. It makes use of six equally sized intervals, with lighter colour shadings indicating more elastic import demand and darker shading pointing towards less elastic or inelastic demand. On the American continent, the United States and Brazil stand out showing the most elastic import demand in North and South America, respectively. Europe is throughout shaded in dark blue with particularly inelastic demand found for Eastern European countries and the Iberian Peninsula. Looking at Asia and Oceania, India and Japan clearly stand out as the countries with the most elastic demand for imports. To the south of the equator, African countries' imports seem to respond only little to price changes. To the north of the equator, however, the picture is very diverse. Grey areas indicate that due to missing data we were not able to compute import demand elasticities for these countries, which are mainly found in Africa and Central Asia.

\section{Figure 3 / Simple average binding elasticities per country}

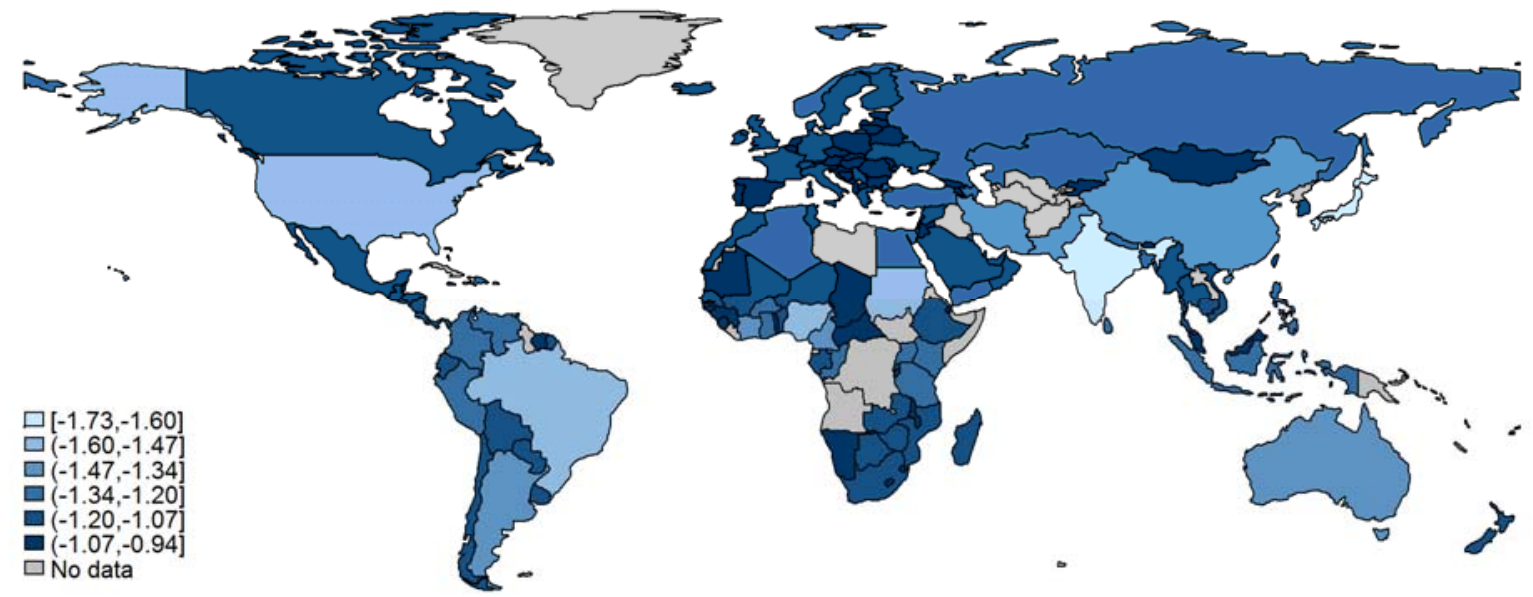

Binding elasticities refer to estimates significantly different from zero at the $10 \%$ level.

Table 2 presents regional average elasticities. The first column shows the results, when we compute the simple average over all HS 6-digit products for each importing country in our sample and further average these results over all countries associated with one geographical region ${ }^{4}$. In the second column, using

4 s. a. $\hat{\varepsilon}_{n n R}=\sum_{i} \frac{\sum_{n} \hat{\varepsilon}_{n n i}}{N} / I, \forall i \in R$; computed over $N$ HS 6-digit products imported by $I$ countries belonging to region $R$. 
Wiiw Working Paper 132

the import-weighted averages, we acknowledge the fact that economically unimportant products might bias our results: Products that are less important for an importer are expected to show lower import values and to face a more volatile demand. We therefore impose import weights on each product for every importer and then take the simple average over all countries to derive a regional average figure. ${ }^{5}$ The third column puts more emphasis on the economically most important countries in the region, by computing an import-weighted average over all importing countries per region. ${ }^{6}$ Columns 4 and 5 indicate how many countries of our sample are assigned to a particular region and how economically developed the region is, approximated by GDP per capita in purchasing power parities, respectively.

Table 1 / Elasticities by importer

\begin{tabular}{|c|c|c|c|c|c|c|c|}
\hline & & s.a. $\hat{\varepsilon}_{n n i}$ & $\begin{array}{l}\text { Binding } \\
\text { s.a. } \hat{\varepsilon}_{n n i}\end{array}$ & $\begin{array}{l}\text { Binding } \\
\text { w.a. } \hat{\varepsilon}_{n n i}\end{array}$ & $\begin{array}{l}\text { Binding } \\
\text { w.a. } \hat{\varepsilon}_{n n i}\end{array}$ & $\begin{array}{l}\text { Binding } \\
\text { w.a. } \hat{\varepsilon}_{n n i}\end{array}$ & \\
\hline ISO2 & Country name & Total & Total & Total & Agri-food & Manufacturing & $\begin{array}{r}\text { GDP } \\
\text { p.c. }\end{array}$ \\
\hline $\mathrm{AL}$ & Albania & -1.045 & -1.049 & -0.961 & -0.967 & -0.959 & 6.7 \\
\hline $\mathrm{DZ}$ & Algeria & -1.472 & -1.310 & -0.951 & -0.978 & -0.944 & 10.5 \\
\hline AG & Antigua and Barbuda & -0.958 & -0.987 & -0.984 & -0.980 & -0.986 & 17.6 \\
\hline AR & Argentina & -1.757 & -1.457 & -0.975 & -1.040 & -0.973 & 15.9 \\
\hline AM & Armenia & -1.047 & -1.063 & -0.968 & -0.966 & -0.969 & 5.2 \\
\hline AW & Aruba & -0.969 & -0.986 & -0.974 & -0.991 & -0.966 & 42.1 \\
\hline$A U$ & Australia & -1.494 & -1.339 & -0.931 & -0.945 & -0.930 & 38.6 \\
\hline AT & Austria & -1.106 & -1.053 & -0.957 & -0.951 & -0.958 & 39.1 \\
\hline$A Z$ & Azerbaijan & -1.431 & -1.332 & -0.949 & -0.964 & -0.947 & 7.5 \\
\hline BS & Bahamas & -1.238 & -1.155 & -0.979 & -0.969 & -0.981 & 25.5 \\
\hline $\mathrm{BH}$ & Bahrain & -1.203 & -1.133 & -1.006 & -0.956 & -1.011 & 31.3 \\
\hline BD & Bangladesh & -1.635 & -1.336 & -0.985 & -0.992 & -0.983 & 1.8 \\
\hline BB & Barbados & -1.049 & -1.051 & -0.969 & -0.963 & -0.970 & 19.6 \\
\hline BY & Belarus & -1.079 & -1.050 & -1.005 & -0.957 & -1.010 & 11.9 \\
\hline BE & Belgium & -0.990 & -0.992 & -0.984 & -0.959 & -0.987 & 36.8 \\
\hline$B Z$ & Belize & -0.964 & -0.972 & -0.989 & -0.972 & -0.992 & 7.4 \\
\hline BJ & Benin & -1.162 & -1.173 & -0.974 & -0.982 & -0.970 & 1.6 \\
\hline BM & Bermuda & -1.100 & -1.100 & -0.963 & -0.950 & -0.966 & 48.7 \\
\hline BT & Bhutan & -0.942 & -0.969 & -0.993 & -0.993 & -0.993 & 5.2 \\
\hline BO & Bolivia & -1.151 & -1.127 & -0.962 & -0.951 & -0.963 & 4.1 \\
\hline BA & Bosnia and Herzegovina & -1.086 & -1.058 & -0.973 & -0.976 & -0.972 & 6.8 \\
\hline BW & Botswana & -1.140 & -1.089 & -0.982 & -0.951 & -0.986 & 11.4 \\
\hline BR & Brazil & -1.903 & -1.572 & -1.002 & -1.017 & -1.002 & 10.7 \\
\hline $\mathrm{BN}$ & Brunei Darussalam & -1.262 & -1.212 & -0.957 & -0.952 & -0.958 & 61.0 \\
\hline BG & Bulgaria & -1.022 & -1.009 & -0.979 & -0.952 & -0.981 & 11.9 \\
\hline BF & Burkina Faso & -1.276 & -1.206 & -0.969 & -0.970 & -0.968 & 1.2 \\
\hline $\mathrm{BI}$ & Burundi & -0.967 & -0.994 & -0.982 & -0.999 & -0.977 & 0.6 \\
\hline$C V$ & Cabo Verde & -0.954 & -0.977 & -0.974 & -0.981 & -0.972 & 4.4 \\
\hline $\mathrm{KH}$ & Cambodia & -1.194 & -1.173 & -0.984 & -0.994 & -0.983 & 1.9 \\
\hline $\mathrm{CM}$ & Cameroon & -1.536 & -1.446 & -0.960 & -0.980 & -0.955 & 2.3 \\
\hline CA & Canada & -1.228 & -1.145 & -0.951 & -0.953 & -0.950 & 38.6 \\
\hline CF & Central African Republic & -0.919 & -1.003 & -0.956 & -0.968 & -0.952 & 0.9 \\
\hline
\end{tabular}

$5 \quad$ country w.a. $=\sum_{i}\left[\sum_{n} \frac{\hat{\varepsilon}_{n n i} \text { Imports }_{i n}}{\text { Imports }_{i}}\right] / I, \forall i \in R$

$6 \quad$ regional w.a. $=\sum_{i}\left[\sum_{n} \frac{\hat{\varepsilon}_{n n i} \text { Import }_{\text {In }}}{\text { Imports }_{i}}\right] / \frac{\text { Imports }_{i}}{\text { Imports }_{R}}, \forall i \in R$ 
Table $1 /$ ctd.

\begin{tabular}{|c|c|c|c|c|c|c|c|}
\hline & & s.a. $\hat{\varepsilon}_{n n i}$ & $\begin{array}{l}\text { Binding } \\
\text { s.a. } \hat{\varepsilon}_{n n i}\end{array}$ & $\begin{array}{l}\text { Binding } \\
\text { w.a. } \hat{\varepsilon}_{n n i}\end{array}$ & $\begin{array}{l}\text { Binding } \\
\text { w.a. } \hat{\varepsilon}_{n n i}\end{array}$ & $\begin{array}{l}\text { Binding } \\
\text { w.a. } \hat{\varepsilon}_{n n i}\end{array}$ & \\
\hline ISO2 & Country name & Total & Total & Total & Agri-food & Manufacturing & $\begin{array}{l}\text { GDP } \\
\text { p.c. }\end{array}$ \\
\hline TD & Chad & -0.865 & -0.941 & -0.965 & -0.968 & -0.964 & 1.5 \\
\hline $\mathrm{CL}$ & Chile & -1.286 & -1.157 & -0.963 & -0.979 & -0.961 & 14.8 \\
\hline $\mathrm{CN}$ & China & -1.621 & -1.389 & -0.966 & -1.049 & -0.963 & 6.8 \\
\hline HK & China, Hong Kong SAR & -1.240 & -1.129 & -1.023 & -0.972 & -1.025 & 41.8 \\
\hline MO & China, Macao SAR & -1.289 & -1.265 & -0.978 & -0.968 & -0.979 & 58.1 \\
\hline $\mathrm{CO}$ & Colombia & -1.524 & -1.326 & -0.956 & -0.995 & -0.951 & 8.8 \\
\hline KM & Comoros & -0.884 & -0.949 & -0.981 & -0.994 & -0.973 & 1.6 \\
\hline CG & Congo & -1.289 & -1.224 & -1.037 & -0.969 & -1.043 & 2.9 \\
\hline CR & Costa Rica & -1.148 & -1.110 & -0.967 & -0.963 & -0.968 & 11.0 \\
\hline $\mathrm{Cl}$ & Côte d'Ivoire & -1.475 & -1.368 & -0.984 & -0.976 & -0.986 & 2.5 \\
\hline HR & Croatia & -1.096 & -1.064 & -0.963 & -0.954 & -0.964 & 17.0 \\
\hline CY & Cyprus & -1.169 & -1.114 & -0.974 & -0.966 & -0.975 & 29.2 \\
\hline$C Z$ & Czech Republic & -1.048 & -1.023 & -0.967 & -0.948 & -0.968 & 24.8 \\
\hline DK & Denmark & -1.219 & -1.120 & -0.948 & -0.969 & -0.945 & 38.2 \\
\hline DJ & Djibouti & -0.938 & -0.955 & -0.987 & -0.991 & -0.986 & 2.6 \\
\hline DM & Dominica & -0.922 & -0.947 & -0.980 & -0.980 & -0.980 & 9.1 \\
\hline DO & Dominican Republic & -1.340 & -1.224 & -0.964 & -0.958 & -0.965 & 9.0 \\
\hline EC & Ecuador & -1.248 & -1.134 & -0.957 & -0.955 & -0.957 & 7.2 \\
\hline EG & Egypt & -1.360 & -1.220 & -0.971 & -0.983 & -0.967 & 6.4 \\
\hline SV & El Salvador & -1.124 & -1.080 & -0.969 & -0.967 & -0.970 & 4.1 \\
\hline EE & Estonia & -1.017 & -1.003 & -0.975 & -0.979 & -0.975 & 17.8 \\
\hline ET & Ethiopia & -1.227 & -1.191 & -0.974 & -0.994 & -0.972 & 0.8 \\
\hline FJ & Fiji & -1.010 & -1.040 & -1.005 & -0.974 & -1.011 & 6.6 \\
\hline $\mathrm{FI}$ & Finland & -1.274 & -1.166 & -0.963 & -0.971 & -0.962 & 35.1 \\
\hline $\mathrm{FR}$ & France & -1.089 & -1.075 & -0.966 & -0.978 & -0.964 & 33.8 \\
\hline GA & Gabon & -1.184 & -1.184 & -0.949 & -0.949 & -0.949 & 12.2 \\
\hline GM & Gambia & -0.945 & -0.981 & -1.004 & -0.993 & -1.010 & 1.6 \\
\hline GE & Georgia & -1.116 & -1.061 & -0.963 & -0.963 & -0.963 & 5.1 \\
\hline DE & Germany & -1.168 & -1.103 & -0.950 & -0.980 & -0.947 & 37.0 \\
\hline $\mathrm{GH}$ & Ghana & -1.360 & -1.264 & -0.959 & -0.968 & -0.958 & 2.6 \\
\hline EL & Greece & -1.223 & -1.128 & -0.979 & -0.969 & -0.980 & 26.2 \\
\hline GD & Grenada & -0.921 & -0.944 & -0.980 & -0.972 & -0.982 & 9.0 \\
\hline GT & Guatemala & -1.152 & -1.084 & -0.975 & -0.961 & -0.977 & 5.4 \\
\hline GN & Guinea & -1.076 & -1.061 & -0.985 & -0.987 & -0.985 & 1.4 \\
\hline GW & Guinea-Bissau & -0.936 & -0.992 & -0.997 & -1.006 & -0.988 & 1.4 \\
\hline $\mathrm{HN}$ & Honduras & -1.100 & -1.075 & -0.986 & -0.968 & -0.990 & 3.7 \\
\hline $\mathrm{HU}$ & Hungary & -1.061 & -1.041 & -0.966 & -0.952 & -0.967 & 19.2 \\
\hline IS & Iceland & -1.177 & -1.105 & -0.974 & -0.953 & -0.976 & 39.3 \\
\hline IN & India & -1.990 & -1.649 & -0.983 & -0.978 & -0.983 & 3.0 \\
\hline ID & Indonesia & -1.443 & -1.277 & -0.979 & -0.981 & -0.979 & 5.6 \\
\hline IR & Iran (Islamic Republic of) & -1.632 & -1.384 & -0.969 & -0.998 & -0.963 & 11.9 \\
\hline IE & Ireland & -1.211 & -1.113 & -0.965 & -0.961 & -0.966 & 39.6 \\
\hline IL & Israel & -1.326 & -1.194 & -0.943 & -0.958 & -0.941 & 30.9 \\
\hline IT & Italy & -1.138 & -1.099 & -0.958 & -0.969 & -0.957 & 33.7 \\
\hline JM & Jamaica & -1.124 & -1.114 & -1.001 & -0.972 & -1.006 & 6.6 \\
\hline$J P$ & Japan & -1.986 & -1.733 & -0.977 & -0.990 & -0.975 & 34.0 \\
\hline
\end{tabular}


Wiiw Working Paper 132

Table $1 /$ ctd.

\begin{tabular}{|c|c|c|c|c|c|c|c|}
\hline & & s.a. $\hat{\varepsilon}_{n n i}$ & $\begin{array}{l}\text { Binding } \\
\text { s.a. } \hat{\varepsilon}_{n n i}\end{array}$ & $\begin{array}{l}\text { Binding } \\
\text { w.a. } \hat{\varepsilon}_{n n i}\end{array}$ & $\begin{array}{l}\text { Binding } \\
\text { w.a. } \hat{\varepsilon}_{n n i}\end{array}$ & $\begin{array}{l}\text { Binding } \\
\text { w.a. } \hat{\varepsilon}_{n n i}\end{array}$ & \\
\hline ISO2 & Country name & Total & Total & Total & Agri-food & Manufacturing & $\begin{array}{r}\text { GDP } \\
\text { p.c. }\end{array}$ \\
\hline jO & Jordan & -1.070 & -1.041 & -0.989 & -0.977 & -0.992 & 6.4 \\
\hline $\mathrm{KZ}$ & Kazakhstan & -1.444 & -1.267 & -0.946 & -0.927 & -0.948 & 12.1 \\
\hline KE & Kenya & -1.275 & -1.209 & -0.965 & -0.986 & -0.963 & 2.2 \\
\hline $\mathrm{KW}$ & Kuwait & -1.429 & -1.244 & -0.934 & -0.942 & -0.933 & 56.3 \\
\hline KG & Kyrgyzstan & -0.988 & -0.998 & -0.985 & -0.977 & -0.986 & 2.6 \\
\hline LV & Latvia & -1.038 & -1.025 & -0.966 & -0.972 & -0.965 & 15.1 \\
\hline LB & Lebanon & -1.168 & -1.123 & -0.983 & -0.968 & -0.986 & 11.0 \\
\hline LS & Lesotho & -0.952 & -0.985 & -0.992 & -0.986 & -0.994 & 1.9 \\
\hline LT & Lithuania & -1.056 & -1.023 & -0.991 & -0.971 & -0.994 & 16.6 \\
\hline LU & Luxembourg & -1.301 & -1.155 & -0.973 & -0.969 & -0.974 & 75.4 \\
\hline MG & Madagascar & -1.099 & -1.092 & -0.964 & -0.994 & -0.959 & 0.9 \\
\hline MW & Malawi & -1.049 & -1.061 & -0.961 & -0.969 & -0.960 & 1.0 \\
\hline MY & Malaysia & -1.090 & -1.069 & -0.989 & -0.959 & -0.991 & 16.4 \\
\hline MV & Maldives & -0.976 & -0.973 & -0.999 & -0.980 & -1.003 & 10.2 \\
\hline ML & Mali & -1.114 & -1.088 & -0.982 & -0.968 & -0.985 & 1.2 \\
\hline MT & Malta & -1.099 & -1.067 & -1.024 & -0.968 & -1.029 & 25.2 \\
\hline MR & Mauritania & -1.009 & -1.042 & -1.000 & -0.989 & -1.002 & 2.5 \\
\hline MU & Mauritius & -1.053 & -1.051 & -0.980 & -0.974 & -0.981 & 14.4 \\
\hline $\mathrm{MX}$ & Mexico & -1.301 & -1.169 & -0.952 & -0.983 & -0.950 & 13.1 \\
\hline MN & Mongolia & -1.044 & -1.045 & -0.979 & -0.963 & -0.981 & 5.4 \\
\hline ME & Montenegro & -1.026 & -1.023 & -0.977 & -0.982 & -0.976 & 10.2 \\
\hline MS & Montserrat & -0.934 & -0.948 & -1.012 & -0.982 & -1.019 & 17.5 \\
\hline MA & Morocco & -1.252 & -1.126 & -0.966 & -0.990 & -0.962 & 5.3 \\
\hline $\mathrm{MZ}$ & Mozambique & -1.183 & -1.136 & -0.959 & -0.972 & -0.957 & 0.8 \\
\hline MM & Myanmar & -1.120 & -1.114 & -0.986 & -0.971 & -0.988 & 2.3 \\
\hline NA & Namibia & -1.079 & -1.071 & -0.968 & -0.963 & -0.969 & 7.0 \\
\hline NP & Nepal & -1.263 & -1.226 & -0.976 & -0.978 & -0.976 & 1.5 \\
\hline $\mathrm{NL}$ & Netherlands & -1.068 & -1.061 & -0.969 & -0.957 & -0.970 & 41.1 \\
\hline NZ & New Zealand & -1.217 & -1.128 & -0.944 & -0.929 & -0.945 & 29.2 \\
\hline $\mathrm{NI}$ & Nicaragua & -1.082 & -1.086 & -0.979 & -0.969 & -0.981 & 3.6 \\
\hline $\mathrm{NE}$ & Niger & -1.243 & -1.186 & -0.961 & -0.986 & -0.954 & 0.8 \\
\hline NG & Nigeria & -1.805 & -1.513 & -0.954 & -0.975 & -0.949 & 2.8 \\
\hline NO & Norway & -1.385 & -1.210 & -0.949 & -0.968 & -0.947 & 49.3 \\
\hline OM & Oman & -1.283 & -1.166 & -0.970 & -0.964 & -0.971 & 28.5 \\
\hline PK & Pakistan & -1.745 & -1.452 & -0.985 & -0.990 & -0.985 & 3.4 \\
\hline PA & Panama & -1.219 & -1.152 & -0.966 & -0.962 & -0.967 & 12.0 \\
\hline PY & Paraguay & -1.224 & -1.160 & -0.975 & -0.962 & -0.976 & 5.3 \\
\hline PE & Peru & -1.458 & -1.276 & -0.954 & -0.958 & -0.954 & 7.1 \\
\hline $\mathrm{PH}$ & Philippines & -1.389 & -1.276 & -0.986 & -0.962 & -0.988 & 4.8 \\
\hline PL & Poland & -1.050 & -1.035 & -0.952 & -0.961 & -0.952 & 16.8 \\
\hline PT & Portugal & -1.090 & -1.044 & -0.955 & -0.965 & -0.954 & 24.0 \\
\hline QA & Qatar & -1.390 & -1.202 & -0.953 & -0.950 & -0.954 & 88.7 \\
\hline $\mathrm{KR}$ & Republic of Korea & -1.307 & -1.186 & -0.981 & -1.002 & -0.980 & 27.0 \\
\hline MD & Republic of Moldova & -0.991 & -0.998 & -0.982 & -0.973 & -0.984 & 2.9 \\
\hline RO & Romania & -1.075 & -1.048 & -0.951 & -0.961 & -0.950 & 12.0 \\
\hline $\mathrm{RU}$ & Russian Federation & -1.544 & -1.334 & -0.915 & -0.959 & -0.907 & 15.2 \\
\hline RW & Rwanda & -1.099 & -1.087 & -0.945 & -0.967 & -0.940 & 1.0 \\
\hline
\end{tabular}


Table $1 /$ ctd.

\begin{tabular}{|c|c|c|c|c|c|c|c|}
\hline & & s.a. $\hat{\varepsilon}_{n n i}$ & $\begin{array}{l}\text { Binding } \\
\text { s.a. } \hat{\varepsilon}_{n n i}\end{array}$ & $\begin{array}{l}\text { Binding } \\
\text { w.a. } \hat{\varepsilon}_{n n i}\end{array}$ & $\begin{array}{l}\text { Binding } \\
\text { w.a. } \hat{\varepsilon}_{n n i}\end{array}$ & $\begin{array}{l}\text { Binding } \\
\text { w.a. } \hat{\varepsilon}_{n n i}\end{array}$ & \\
\hline ISO2 & Country name & Total & Total & Total & Agri-food & Manufacturing & $\begin{array}{r}\text { GDP } \\
\text { p.c. }\end{array}$ \\
\hline $\mathrm{KN}$ & Saint Kitts and Nevis & -0.928 & -0.947 & -0.968 & -0.977 & -0.966 & 17.2 \\
\hline LC & Saint Lucia & -0.942 & -0.965 & -0.994 & -0.980 & -0.998 & 8.9 \\
\hline ST & Sao Tome and Principe & -0.897 & -0.930 & -1.001 & -0.996 & -1.002 & 2.2 \\
\hline SA & Saudi Arabia & -1.343 & -1.186 & -0.935 & -0.943 & -0.934 & 29.7 \\
\hline SN & Senegal & -1.140 & -1.133 & -0.981 & -0.988 & -0.978 & 2.0 \\
\hline RS & Serbia & -1.114 & -1.078 & -0.960 & -0.947 & -0.961 & 9.4 \\
\hline SC & Seychelles & -0.931 & -0.957 & -1.055 & -1.017 & -1.063 & 20.0 \\
\hline SL & Sierra Leone & -1.003 & -1.021 & -1.044 & -0.977 & -1.052 & 1.3 \\
\hline SG & Singapore & -1.085 & -1.044 & -1.045 & -0.960 & -1.048 & 51.5 \\
\hline SK & Slovakia & -1.029 & -1.027 & -0.975 & -0.951 & -0.976 & 19.7 \\
\hline SI & Slovenia & -1.051 & -1.026 & -0.968 & -0.957 & -0.969 & 25.6 \\
\hline ZA & South Africa & -1.275 & -1.175 & -0.952 & -0.985 & -0.950 & 10.2 \\
\hline ES & Spain & -1.093 & -1.064 & -0.954 & -0.960 & -0.954 & 29.6 \\
\hline LK & Sri Lanka & -1.287 & -1.225 & -0.972 & -0.983 & -0.970 & 5.9 \\
\hline VC & St. Vincent and the Grenadines & -0.918 & -0.949 & -0.984 & -0.978 & -0.986 & 8.2 \\
\hline PS & State of Palestine & -1.153 & -1.137 & -1.003 & -0.981 & -1.010 & 3.7 \\
\hline SD & Sudan (Former) & -1.719 & -1.503 & -0.948 & -0.966 & -0.945 & 2.5 \\
\hline SR & Suriname & -1.001 & -1.005 & -0.979 & -0.966 & -0.981 & 10.1 \\
\hline Sz & Swaziland & -0.971 & -0.988 & -0.986 & -0.973 & -0.988 & 7.6 \\
\hline SE & Sweden & -1.243 & -1.158 & -0.963 & -0.960 & -0.964 & 38.3 \\
\hline $\mathrm{CH}$ & Switzerland & -1.234 & -1.122 & -1.027 & -0.953 & -1.030 & 46.6 \\
\hline SY & Syrian Arab Republic & -1.273 & -1.169 & -0.994 & -0.974 & -0.998 & 3.3 \\
\hline TW & Taiwan & -1.277 & -1.168 & -0.985 & -0.967 & -0.985 & 35.3 \\
\hline MK & TFYR of Macedonia & -0.996 & -1.019 & -0.977 & -0.964 & -0.978 & 9.3 \\
\hline $\mathrm{TH}$ & Thailand & -1.229 & -1.160 & -0.978 & -0.959 & -0.979 & 10.1 \\
\hline TG & Togo & -0.972 & -0.998 & -0.984 & -0.970 & -0.986 & 1.2 \\
\hline TT & Trinidad and Tobago & -1.195 & -1.108 & -0.999 & -0.964 & -1.002 & 20.5 \\
\hline TN & Tunisia & -1.148 & -1.102 & -0.969 & -0.976 & -0.968 & 9.0 \\
\hline TR & Turkey & -1.338 & -1.224 & -0.956 & -0.988 & -0.955 & 13.6 \\
\hline TC & Turks and Caicos Islands & -0.902 & -0.941 & -1.005 & -0.983 & -1.012 & 20.2 \\
\hline TZ & U.R. of Tanzania: Mainland & -1.327 & -1.278 & -0.991 & -0.986 & -0.992 & 1.5 \\
\hline UG & Uganda & -1.388 & -1.287 & -0.965 & -0.992 & -0.961 & 1.4 \\
\hline UA & Ukraine & -1.251 & -1.148 & -0.979 & -0.951 & -0.981 & 7.2 \\
\hline $\mathrm{AE}$ & United Arab Emirates & -1.269 & -1.158 & -0.963 & -0.950 & -0.964 & 79.0 \\
\hline UK & United Kingdom & -1.150 & -1.107 & -0.961 & -0.973 & -0.959 & 36.1 \\
\hline US & United States & -1.717 & -1.534 & -0.997 & -1.043 & -0.995 & 47.8 \\
\hline UY & Uruguay & -1.260 & -1.199 & -0.975 & -0.953 & -0.977 & 13.3 \\
\hline VE & Venezuela & -1.419 & -1.297 & -0.930 & -0.971 & -0.923 & 10.6 \\
\hline VN & Viet Nam & -1.152 & -1.091 & -0.974 & -0.971 & -0.974 & 3.1 \\
\hline YE & Yemen & -1.394 & -1.287 & -0.980 & -0.985 & -0.977 & 2.4 \\
\hline ZM & Zambia & -1.161 & -1.133 & -0.960 & -0.948 & -0.961 & 2.0 \\
\hline ZW & Zimbabwe & -1.082 & -1.082 & -0.984 & -0.973 & -0.986 & 2.3 \\
\hline
\end{tabular}

Note: s.a. refers to the simple average over all HS 6-digit products per country: $\sum_{h} \hat{\varepsilon}_{n n i} / H$. w.a. refers to the import-weighted average per country: $\sum_{h}\left(\hat{\varepsilon}_{n n i} x\right.$ Imports $_{i h} /$ Imports $\left._{i}\right)$. Binding elasticities refer to estimates significantly different from zero at the $10 \%$ level. GDP p.c. refers to the average expenditure-side real GDP per capita measured at chained PPPs in thousand 2011 USD for the period 1995-2014. 
Looking at simple average elasticities, it seems that the second poorest region and the richest region in the world, i.e. South Asia and North America, are associated with the most elastic demand, while the least elastic import demand is found for the poorest and the second richest region in the world, i.e. Sub-Saharan Africa as well as Europe and Central Asia. Admittedly, the region Europe and Central Asia according to the World Bank List of Economies is the largest and probably most diverse region in our sample. Yet, even restricting our view to the European Union, import demand is much less elastic than demand of the United States (see Figure 4).

Employing import weights on world regions, Table 2 draws a picture of overall inelastic demand for every world region, greatly diminishing regional differences. It should be noted, however, that if demand is highly price-elastic, trade volumes might be scaled down considerably and country averages of elasticities could give the wrong picture of too low elasticities.

\section{Table 2 / Regional elasticities}

\begin{tabular}{|c|c|c|c|c|c|}
\hline Elasticities per region & $\begin{array}{c}\text { Simple } \\
\text { avg. }\end{array}$ & $\begin{array}{c}\text { Country } \\
\text { w.a. }\end{array}$ & $\begin{array}{c}\text { Regional } \\
\text { w.a. }\end{array}$ & $\begin{array}{c}\text { No. of } \\
\text { countries }\end{array}$ & $\begin{array}{c}\text { GDP p.c. } \\
\text { (PPP) }\end{array}$ \\
\hline Europe and Central Asia & -1.14 & -0.95 & -0.94 & 46 & 23.8 \\
\hline Latin America and the Caribbean & -1.17 & -0.96 & -0.94 & 33 & 10.9 \\
\hline East Asia \& Pacific & -1.29 & -0.97 & -0.97 & 19 & 9.6 \\
\hline Middle East and North Africa & -1.27 & -0.96 & -0.95 & 19 & 12.5 \\
\hline Sub-Saharan Africa & -1.15 & -0.96 & -0.95 & 41 & 2.6 \\
\hline
\end{tabular}

Note: Simple avg. refers to the simple average computed over all country averages per region. Country w.a. refers to the simple average over country-specific import-weighted elasticities per region. Regional w.a. refers to the import-weighted average over country-specific import-weighted elasticities. GDP p.c. refers to the regional average expenditure-side real GDP per capita measured at chained PPPs in thousand 2011 USD for the period 1995-2014. Please refer to the Appendix for the categorisation of our country sample according to the World Bank List of Economies (July 2015).

Figure 4 further elaborates on country differences by plotting importer-specific import demand elasticities against GDP and GDP per capita at purchasing power parities (PPP), respectively. Note that we opted for showing GDP per capita in log scales, i.e. the difference between two ticks on the $x$-axis indicates a doubling of income at PPP.

As we have already observed looking at the world map, the countries with the highest simple average elasticities in absolute terms - Japan, India, Brazil, the United States, Nigeria - belong with the exception of Japan to the most populous countries in their respective regions. They are associated with the economically most important countries in the region, but the difference in GDP per capita between these countries is huge. On the other end of the spectrum, the ten countries associated with the lowest import demand elasticities are small island states, with the exception of landlocked and poverty- and violence-ridden Chad.

The most intuitive interpretation would be, that both physically larger and economically more developed countries can more easily substitute imported products by domestically produced goods, whereas small island states and poor countries lack the capacities of developing and maintaining a diverse set of domestic industries and are more dependent on imports. This assumption is in line with the finding that the picture reverses when focusing on the most important traded commodities in terms of trade volumes 
by attaching import weights to every HS 6-digit product within a country. We find that bigger economies are associated with a lower import-weighted average import elasticity. For imported products which can be substituted by domestically produced goods, we would expect that import demand is more elastic and that trade volumes are lower compared to products, which are not produced domestically. Employing import-weights therefore would scale down elasticities of products facing domestic competition and puts more emphasis on products for which countries are more dependent on imports.

By contrast, looking at the overall picture of the right panel of Figure 4 does not allow to assume that richer countries are associated with more or less elastic demand. However, focusing on the sub-sample of Members States of the European Union a trend towards more elastic demand for richer countries is visible, which is not only a matter of the absolute size of the economy.

\section{Figure 4 I Binding elasticities over income}
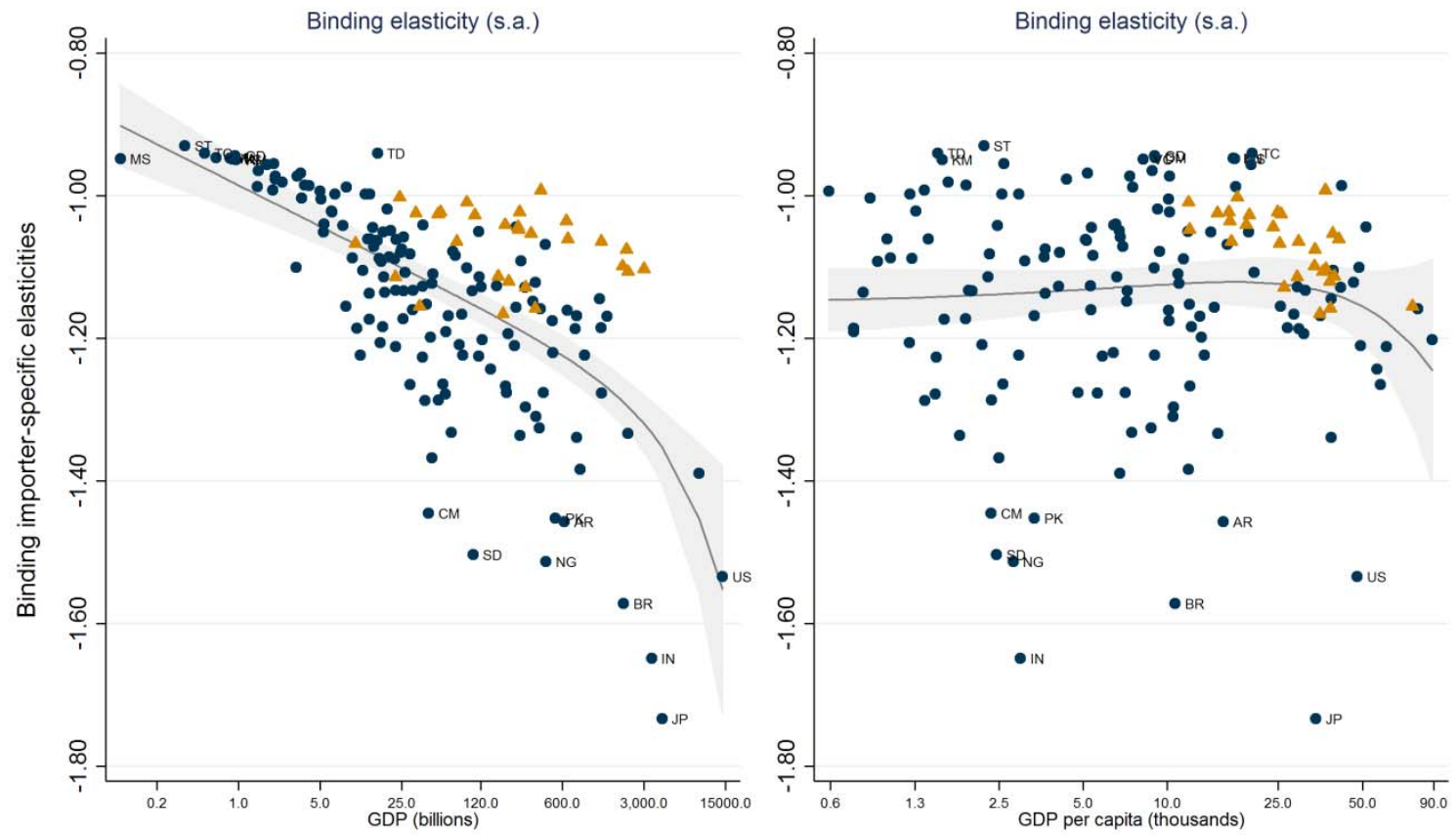

Note: s.a. refers to the simple average per country computed over all HS 6-digit products: $\sum_{n} \hat{\varepsilon}_{n n i} / N$. Binding elasticities refer to estimates significantly different from zero at the $10 \%$ level. GDP p.c. refers to the average expenditure-side real GDP per capita per country measured at chained PPPs in thousand 2011 USD for the period 1995-2014. EU Member States highlighted as orange triangles. The fitted line stems from a second order fractional polynomial estimation of binding elasticities on GDP per capita.

Table 3 summarises our previously discussed possible determinants of import demand elasticities by regressing binding importer- and product-specific elasticities on country characteristics. We find a higher share of the imported good $n$ in GDP to be associated with a less elastic demand. Economically and physically bigger economies, captured by GDP and its surface area, show significantly higher (i.e. more negative) import demand elasticities. We approximate a country's status of development by three different measures. These three measures are GDP per capita, the Human Development Index (HDI) and the Economic Complexity Index (ECI). In addition to GDP per capita, the HDI published by the United Nations considers the dimensions health and education to describe a country's level of 
wiiw Working Paper 132

development. The ECI provided by the Center for International Development at Harvard University captures how diversified an economy is with respect to the level of complexity of products and the number of products it exports and can be considered as an alternative measure for development (Hausmann et al., 2011). These three measures grasp different dimensions of development but are closely related and do show that demand become less elastic with a higher level of development but that this effect is diminishing. Positive coefficients on the dummy variables for landlocked countries and Small Island Developing States (SIDS) in in line with our expectation that countries that are more dependent on imports exhibit a less elastic import demand. Finally, Table 3 shows that membership to the EU or the WTO is associated with lower price responsiveness, whereas a higher share of fuel exports in GDP points towards more elastic demand.

Table 3 / Regression of binding import demand elasticities on country characteristics

\begin{tabular}{|c|c|c|c|c|}
\hline & $(1)$ & $(2)$ & (3) & $(4)$ \\
\hline \multirow[t]{2}{*}{ Product's share in GDP } & $9.048^{*+* t+}$ & $5.685^{*+* t}$ & $5.878^{*+*}$ & $4.615^{* *}$ \\
\hline & [1.237] & [1.335] & [1.363] & [1.855] \\
\hline \multirow[t]{2}{*}{ GDP } & $-7.74 \mathrm{e}-08^{* \star *}$ & $-4.42 \mathrm{e}-08^{*+* t}$ & $-4.56 e-08^{\star \star \star}$ & $-4.61 e-08^{\star \star \star}$ \\
\hline & [3.93e-09] & [3.33e-09] & [3.33e-09] & {$[4.01 \mathrm{e}-09]$} \\
\hline \multirow[t]{2}{*}{$(G D P)^{2}$} & $4.20 \mathrm{e}-15^{\star \star \star *}$ & $2.21 \mathrm{e}-15^{\star \star \star \star}$ & $2.28 \mathrm{e}-15^{\star \star \star \star}$ & $2.32 \mathrm{e}-15^{\star \star \star t}$ \\
\hline & {$[3.03 e-16]$} & [2.56e-16] & {$[2.56 \mathrm{e}-16]$} & {$[2.89 \mathrm{e}-16]$} \\
\hline \multirow[t]{2}{*}{ GDP p.c. } & $0.000945^{* t * t}$ & $0.00172^{* \star \star}$ & & \\
\hline & [0.000332] & [0.000282] & & \\
\hline \multirow[t]{2}{*}{$(\text { GDP p.c. })^{2}$} & $-0.0000193^{* * *}$ & $-0.0000267^{\star \star \star}$ & & \\
\hline & [0.00000475] & {$[0.00000401]$} & & \\
\hline \multirow[t]{2}{*}{ HDI } & & & $0.418^{*+* t}$ & \\
\hline & & & [0.0844] & \\
\hline \multirow[t]{2}{*}{$(\mathrm{HDI})^{2}$} & & & $-0.259^{\star * \star *}$ & \\
\hline & & & [0.0663] & \\
\hline \multirow[t]{2}{*}{$\mathrm{ECl}$} & & & & $0.0377^{\star \star \star}$ \\
\hline & & & & {$[0.00273]$} \\
\hline \multirow[t]{2}{*}{$(E C I)^{2}$} & & & & $-0.0230^{\star * *}$ \\
\hline & & & & {$[0.00179]$} \\
\hline \multirow[t]{2}{*}{ Area } & $-8.66 \mathrm{e}-09^{\star \star *}$ & $-6.60 e-09^{* \star * t}$ & $-6.12 \mathrm{e}-09^{\text {tht }}$ & $-6.63 e-09^{\star k \star t}$ \\
\hline & [1.11e-09] & [9.37e-10] & [9.39e-10] & {$[1.03 e-09]$} \\
\hline \multirow[t]{2}{*}{ Landlocked } & $0.0329^{* \star \star}$ & $0.0172^{\star \star \star}$ & $0.0219^{\star * \star}$ & $0.0254^{\star \star *}$ \\
\hline & {$[0.00479]$} & [0.00404] & [0.00413] & {$[0.00522]$} \\
\hline \multirow[t]{2}{*}{ Small Island Developing State } & $0.120^{\star * *}$ & $0.0408^{\star \star \star}$ & $0.0379^{\star \star \star}$ & $0.0178^{* *}$ \\
\hline & {$[0.00577]$} & {$[0.00491]$} & {$[0.00505]$} & {$[0.00874]$} \\
\hline \multirow[t]{2}{*}{ EU membership } & $0.101^{\text {**t }}$ & $0.0819^{\star \star \star}$ & $0.0818^{\star \star \star}$ & $0.0785^{\star \star \star *}$ \\
\hline & {$[0.00541]$} & {$[0.00457]$} & {$[0.00466]$} & {$[0.00557]$} \\
\hline \multirow[t]{2}{*}{ WTO membership } & $0.0139^{* *}$ & $0.0189^{* \star *}$ & $0.0261^{* * *}$ & $0.0292^{* \star *}$ \\
\hline & {$[0.00575]$} & {$[0.00485]$} & {$[0.00527]$} & {$[0.00638]$} \\
\hline \multirow[t]{2}{*}{ Exports of fuels in $\%$ of GDP } & $-0.0307^{\star \star \star *}$ & $-0.0281^{\star * \star}$ & $-0.0353^{* * *}$ & $-0.0167^{\star * *}$ \\
\hline & [0.00568] & {$[0.00479]$} & [0.00461] & [0.00623] \\
\hline \multirow[t]{2}{*}{ Constant } & $-1.155^{\star \star \star}$ & $-1.164^{\star \star \star *}$ & $-1.316^{* \star \star}$ & $-1.159^{\star * \star}$ \\
\hline & [0.00643] & {$[0.00544]$} & [0.0272] & {$[0.00634]$} \\
\hline Observations & 442281 & 442281 & 431369 & 343471 \\
\hline$R^{2}$ & 0.006 & 0.306 & 0.308 & 0.317 \\
\hline Product fixed effects & No & Yes & Yes & Yes \\
\hline
\end{tabular}




\subsection{ELASTICITIES BY PRODUCT CATEGORIES}

In section 4.1 we explored our import demand elasticity results by importer. In this section we seek to further elaborate differences and commonalities along different product groups.

We start off by illustrating how elasticities vary between the agri-food and the manufacturing sectors. Considering first simple averages, we find that for a great majority of countries in our sample, 158 out of 167 , the agri-food sector appears to face a more elastic demand than the manufacturing sector. However, when imposing product-specific import weights - separately for each sector - the import demand for products of the manufacturing sector shows to be more elastic for 91 countries, as opposed to 9 countries without import-weights.

Focusing on import weighted results as reported in columns four and five of Table 1, there is a tendency observable that for countries exhibiting an overall elastic demand, the manufacturing sector is more elastic than the agri-food sector. The top 5 countries with the most elastic total import demand form a very diverse group of countries consisting of the Seychelles, Singapore, Sierra Leone, Congo and Switzerland. By contrast, for countries for which we estimated an overall inelastic demand, imports of the agri-food sector seem to be more price-responsive. The bottom 5 countries, for which the least elastic total import demand was estimated, represent countries rich in natural resources - particularly fossil fuels - led by Russia and followed by Venezuela, Australia, Kuwait and Saudi Arabia.

The ranking of overall import-weighted elasticities is to a great extent dictated by the manufacturing sector. Still, it is worth considering the elasticities for the agricultural sector. The ranking of elasticities for agri-food products from most elastic to inelastic is led by China, the United States and Argentina with import-weighted elasticities of around -1.04. The lowest import demand elasticities for the agri-food sector were evaluated for Kazakhstan and New Zealand, followed by Kuwait, Saudi Arabia and Australia.

As regressions were run separately for every product at the HS 6-digit level, a natural second step is to look at aggregates for the $21 \mathrm{HS}$ sections, with the first four sections representing the agricultural sector. Figure 5 illustrates binding simple average elasticities per section for the European Union, the United States and the rest of the countries in our sample (RoW).

The graph shows, first, that highest import demand elasticities for all three groups can be attributed to animals, meat and fats, as well as mineral and paper products. Vegetable products and prepared foodstuff show more modest elasticity estimates, comparable with textiles or products of the chemical industry. Second, with very few exceptions, import demand of the United States is more elastic than import demand of the European Union. It has to be noted, however, that figures for the EU represent average elasticities over Member States without differentiating between extra- and intra-EU trade. Third, product categories for which import demand is relatively inelastic, i.e. smaller than -1 for every country group, belong to the luxury segment (such as works of arts, peals and precious metals), or concern machinery and electrical equipment and finally arms and ammunition. 


\section{Figure 5 / Binding simple average elasticities per HS Section}

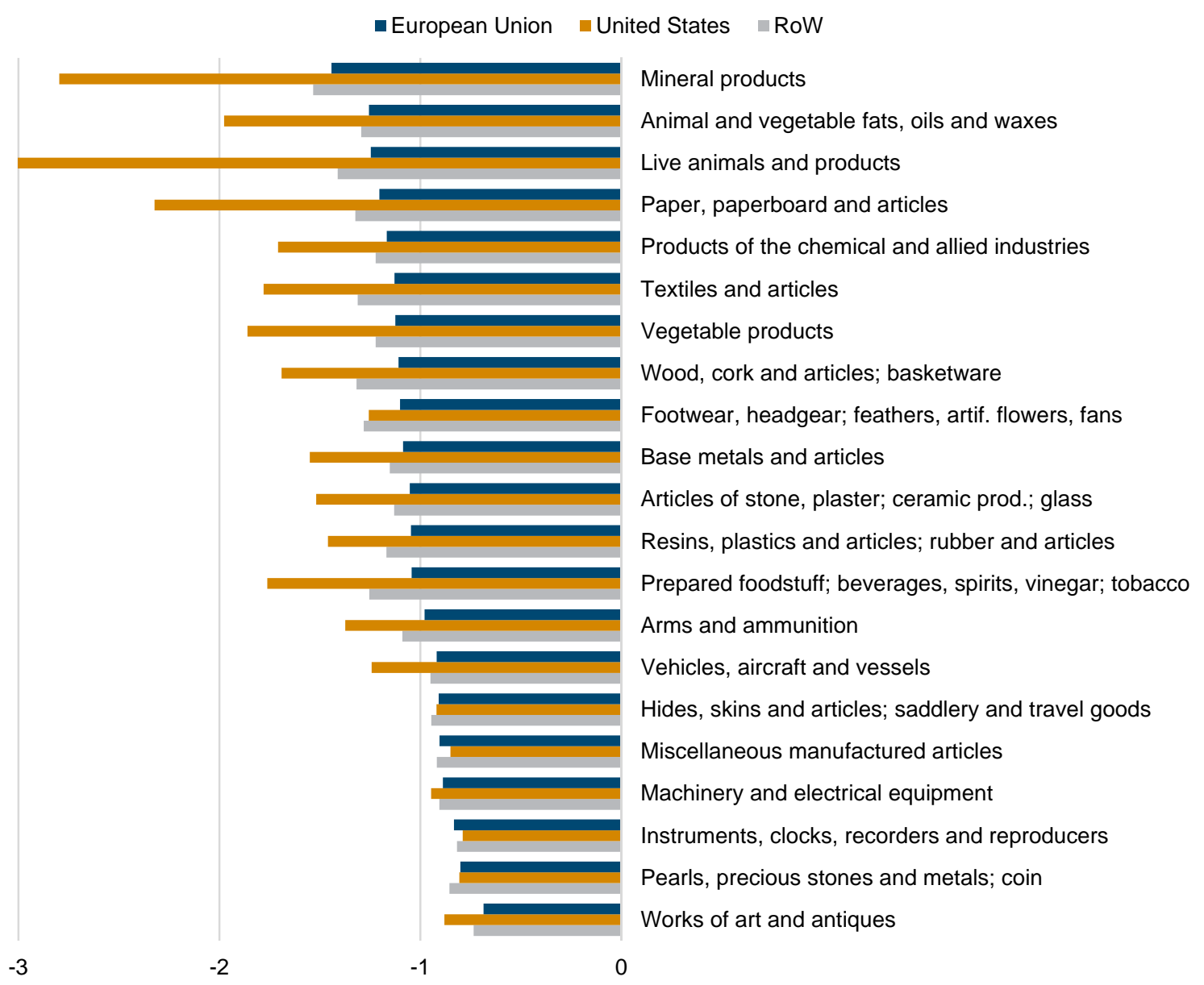

Note: Binding elasticities refer to estimates significantly different from zero at the $10 \%$ level.

Technology seems to be a promising candidate for at least partly explaining this pattern. Using a correspondence from HS 6-digit products to ISIC (International Standard Industrial Classification) 4-digit industries we can differentiate our import demand elasticity results for the manufacturing industries with respect to the OECD technology intensity definition as proposed by Hatzichronoglou (1997). Indeed, simple t-tests reveal that distributions of elasticities are significantly different between various technology intensity groups, with more R\&D content being associated with lower mean and median elasticities in absolute terms. Some manufactured products, as well as products belonging to the agricultural sector, were not assigned to any technology intensity class (low, medium-low, medium-high or high technology intensity). Median elasticities of these products were found to be not significantly different from median import demand elasticities for low-tech manufacturing products.

A different product classification is adopted for input-output tables, as used by the World Input-Output Database (WIOD ${ }^{7}$ ) project (Timmer et al., 2015). Out of 35 sectors currently included in the WIOD database, our data covers seventeen sectors, as our analysis is restricted to trade in goods and does not include trade in services. Table 4 presents our results split up by these sectors.

$7 \quad$ See www.wiod.org 
Table 4 / Elasticities by WIOD sector

\begin{tabular}{|c|c|c|c|c|c|c|c|}
\hline \multirow{2}{*}{ Sector } & & \multicolumn{3}{|c|}{ All Elasticities } & \multicolumn{3}{|c|}{ Binding Elasticities } \\
\hline & & \multirow{2}{*}{$\begin{array}{c}\text { Simple } \\
\text { avg. } \\
-1.376\end{array}$} & \multirow{2}{*}{$\begin{array}{c}\text { Country } \\
\text { w.a. } \\
-0.946\end{array}$} & \multirow{2}{*}{$\begin{array}{c}\text { Sector } \\
\text { w.a. } \\
-0.934\end{array}$} & \multirow{2}{*}{$\begin{array}{c}\text { Simple } \\
\text { avg. } \\
-1.246\end{array}$} & \multirow{2}{*}{$\begin{array}{l}\text { Country } \\
\text { w.a. } \\
-0.959\end{array}$} & \multirow{2}{*}{$\begin{array}{l}\text { Sectol } \\
\text { w.a. } \\
-0.959\end{array}$} \\
\hline c1 & Agriculture, Hunting, Forestry and Fishing & & & & & & \\
\hline c2 & Mining and Quarrying & -1.695 & -1.008 & -1.011 & -1.413 & -1.008 & -1.012 \\
\hline c3 & Food, Beverages and Tobacco & -1.529 & -0.953 & -0.959 & -1.335 & -0.970 & -0.989 \\
\hline c4 & Textiles and Textile Products & -1.411 & -0.986 & -1.004 & -1.310 & -0.997 & -1.017 \\
\hline c5 & Leather, Leather and Footwear & -1.324 & -1.000 & -0.972 & -1.318 & -1.042 & -0.991 \\
\hline c6 & Wood and Products of Wood and Cork & -1.333 & -1.005 & -0.981 & -1.306 & -1.025 & -0.992 \\
\hline c7 & Pulp, Paper, Printing and Publishing & -1.319 & -0.942 & -0.956 & -1.297 & -0.956 & -0.976 \\
\hline c8 & Coke, Refined Petroleum and Nuclear Fuel & -2.347 & -1.178 & -1.306 & -1.876 & -1.167 & -1.305 \\
\hline c9 & Chemicals and Chemical Products & -1.316 & -0.929 & -0.924 & -1.231 & -0.947 & -0.952 \\
\hline c10 & Rubber and Plastics & -0.991 & -0.944 & -0.944 & -1.034 & -0.963 & -0.967 \\
\hline c11 & Other Non-Metallic Mineral & -1.138 & -0.967 & -0.952 & -1.160 & -0.980 & -0.983 \\
\hline c12 & Basic Metals and Fabricated Metal & -1.189 & -0.938 & -0.953 & -1.148 & -0.958 & -0.987 \\
\hline c13 & Machinery, Nec & -0.864 & -0.882 & -0.862 & -0.917 & -0.906 & -0.895 \\
\hline c14 & Electrical and Optical Equipment & -0.817 & -0.840 & -0.884 & -0.851 & -0.874 & -0.911 \\
\hline c15 & Transport Equipment & -0.932 & -0.924 & -0.928 & -0.972 & -0.940 & -0.945 \\
\hline c16 & Manufacturing, Nec; Recycling & -1.054 & -0.906 & -0.887 & -1.032 & -0.919 & -0.902 \\
\hline c17 & Electricity, Gas and Water Supply & -2.649 & -2.636 & -1.868 & -2.035 & -2.051 & -1.868 \\
\hline
\end{tabular}

Note: Simple avg. refers to the simple average computed over all country averages per WIOD sector. Country w.a. refers to the simple average over country-specific import-weighted elasticities per WIOD sector. Sector w.a. refers to the importweighted average over country-specific import-weighted elasticities. Binding elasticities refer to estimates significantly different from zero at the $10 \%$ level.

Independently of the weights employed and whether we consider all estimates or only binding elasticities, the energy sectors, i.e. 'Electricity, Gas and Water Supply' and 'Coke, Refined Petroleum and Nuclear Fuel', surprisingly always appear as the most demand-elastic. Restricting our analysis to HS27 (Mineral fuels, mineral oils and products of their distillation) and considering the pre- and the postcrisis period separately, we do find that demand for goods destined for final consumption was particularly elastic prior to the onset of the global economic crisis, whereas it appeared very priceinelastic also in comparison to mineral products used as intermediate products between 2009 and 2014.

Note, however, that the energy sectors are to a great extent covered by statistics on trade in services, which are not covered by our analysis. The results for 'Electricity, Gas and Water Supply' are based on only 118 estimates for two HS 6-digit products for which commodity trade data is available ${ }^{8}$. The sector 'Coke, Refined Petroleum and Nuclear Fuel' is covered by 39 HS 6-digit products and 3,884 estimates. Other WIOD sectors represent on average 378 HS 6-digit products and 47,389 elasticity estimates.

Simple average elasticities are also high for food, beverages and tobacco, but making use of import weights the sector shifts half-way down the ranking. The sectors for electrical and optical equipment, other machinery and transport equipment feature as the most demand-inelastic sectors.

In addition to sectoral classifications one might expect differences in import demand elasticities with respect to the way they are used in the economy. Imports might be used as (i) final consumption goods, 
(ii) intermediate goods in the production process of final goods, or (iii) by firms in the form of stocks or gross fixed capital formation (GFCF).

This analysis is particularly interesting in today's context of a global trade slowdown, or even 'trade plateau' (Evenett and Fritz, 2016), and negotiations of mega-regional trade deals in which non-tariff measures play a prominent role. Every year during the period 1995-2014 imports of intermediates represented more than $52 \%$ of global imports. The importance of global value chains as exemplified by intermediate goods trade is increasing over time, with only three major setbacks in 1998, in 2009 following the global economic and financial crisis and in 2014.

We borrow a correspondence table that links HS 6-digit products to these three broad categories, with about $15 \%$ of products being reclassified for the WIOD project to account for the fact that some products qualify for more than one category (e.g. HS 940540 electric lamps and lighting fittings).

Table 5 summarises our results for these three categories. It is evident at first sight that intermediate goods face the most elastic demand, followed by final consumption goods, while demand for GFCF goods appears throughout quite inelastic. This result remains unchanged when excluding the energy sector ${ }^{9}$.

Table 5 / Elasticities by product use

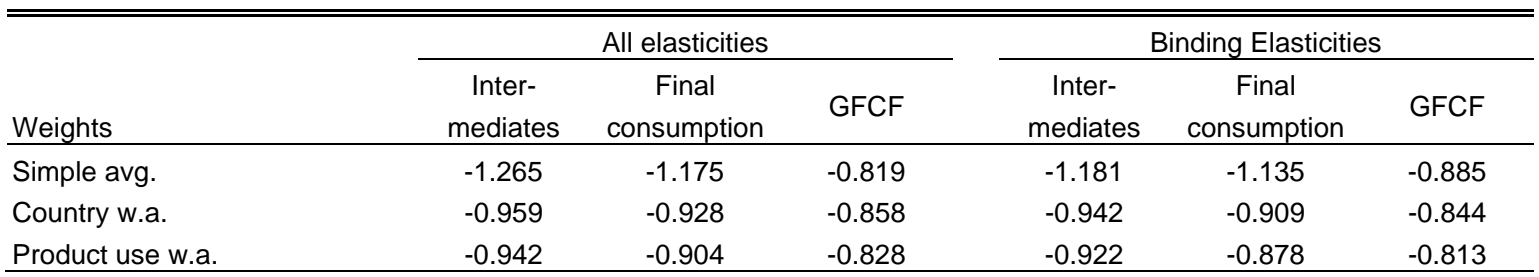

Note: Simple avg. refers to the simple average computed over all country averages per product category of its use. Country w.a. refers to the simple average over country-specific import-weighted elasticities per product category. Product use w.a. refers to the import-weighted average over country-specific import-weighted elasticities. Binding elasticities refer to estimates significantly different from zero at the $10 \%$ level. GFCF refers to Gross Fixed Capital Formation.

Table 6 summarises our discussion on cross-product differences in import demand elasticities. As in Table 3 we find a positive coefficient on a product's share in GDP, however, it becomes non-significant when accounting for importer fixed effects. Other factors that potentially decrease the price elasticity of demand are (i) the technological intensity of a product, (ii) the number of countries exporting a specific product and (iii) the number of importers of a specific product. One argument would be that technologyintensive products cannot easily be substituted by domestic production. The number of exporting countries per product is a proxy for the possibility to substitute between different exporters. The greater the number of suppliers of a specific product, the easier it is for the importing country to substitute imports between different source countries, leaving the share of a product in per cent of GDP unchanged. The number of importers per product might be an indication of the market power of the exporting country. The greater the number of importers of one specific product per exporter, the smaller an importer's bargaining power and its import demand elasticity. 
Negative coefficients are found for the sector dummy, indicating that agri-food products on average face a more elastic import demand. The regression table once more highlights that on average goods contributing to gross fixed capital formation (base line) face the most inelastic demand, followed by final consumption goods and intermediate goods. These findings persist even when fuels (column 3 ) and products without an assigned technology intensity measure (column 4) are excluded from the regression. Differences in import demand elasticities across all these variables are statistically significant. Note, however, that the predictive power of these product characteristics is very limited.

Table 6 / Regression of binding import demand elasticities on product characteristics

\begin{tabular}{|c|c|c|c|c|}
\hline & $(1)$ & (2) & (3) & (4) \\
\hline \multirow[t]{2}{*}{ Product's share in GDP } & $2.722^{* k}$ & 0.957 & 1.360 & -0.265 \\
\hline & [1.211] & [1.206] & [1.839] & [1.815] \\
\hline \multirow[t]{2}{*}{ Sector dummy (1 = agri-food) } & $-0.0628^{\star \star \star}$ & $-0.0677^{\star \star \star}$ & $-0.0837^{\star \star \star}$ & $-0.0870^{* \star \star}$ \\
\hline & {$[0.00647]$} & {$[0.00644]$} & {$[0.00641]$} & {$[0.00697]$} \\
\hline \multirow[t]{2}{*}{ Number of exporters per product } & $0.00270^{\star \star *}$ & $0.00260 * \star \star$ & $0.00251^{\star \star *}$ & $0.00228^{\star \star *}$ \\
\hline & {$[0.000139]$} & {$[0.000139]$} & [0.000138] & {$[0.000140]$} \\
\hline \multirow[t]{2}{*}{ Number of importers per product } & $0.00467^{\star \star \star}$ & 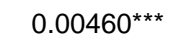 & $0.00455^{\star \star \star}$ & $0.00519 * \star \star$ \\
\hline & {$[0.000146]$} & {$[0.000147]$} & {$[0.000146]$} & {$[0.000151]$} \\
\hline \multirow[t]{2}{*}{ Low-tech } & $-0.0555^{\star \star \star}$ & $-0.0582^{\star \star \star}$ & $-0.0897^{* * *}$ & 0 \\
\hline & {$[0.00771]$} & {$[0.00767]$} & {$[0.00777]$} & [.] \\
\hline \multirow[t]{2}{*}{ Medium-low-tech } & 0.00130 & 0.00647 & $-0.0236^{\star \star \star}$ & $0.0471^{\star \star \star}$ \\
\hline & {$[0.00861]$} & {$[0.00856]$} & {$[0.00868]$} & {$[0.00539]$} \\
\hline \multirow[t]{2}{*}{ Medium-high-tech } & 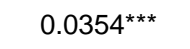 & $0.0461^{\star \star \star}$ & 0.0110 & $0.0850 * \star \star$ \\
\hline & {$[0.00834]$} & {$[0.00830]$} & {$[0.00843]$} & {$[0.00497]$} \\
\hline \multirow[t]{2}{*}{ High-tech } & $0.221^{\star \star \star}$ & $0.225^{\star \star \star}$ & $0.190^{\star \star \star}$ & $0.270^{\star \star \star}$ \\
\hline & {$[0.0101]$} & {$[0.0100]$} & {$[0.0101]$} & [0.00709] \\
\hline \multirow[t]{2}{*}{ Final consumption good } & $-0.0925^{\star \star \star}$ & $-0.0951^{\star \star \star}$ & $-0.0953^{\star \star \star}$ & $-0.119^{\star \star \star}$ \\
\hline & {$[0.00698]$} & {$[0.00696]$} & {$[0.00686]$} & [0.00682] \\
\hline \multirow[t]{2}{*}{ Intermediate good } & $-0.154^{\star \star *}$ & $-0.149^{* \star \star}$ & $-0.150^{* \star \star}$ & $-0.144^{\star \star \star}$ \\
\hline & {$[0.00566]$} & {$[0.00564]$} & {$[0.00556]$} & [0.00543] \\
\hline \multirow[t]{2}{*}{ Constant } & $-1.884^{\star \star \star}$ & $-1.872^{\star \star \star}$ & $-1.822^{\star \star \star}$ & $-1.974^{\star \star \star}$ \\
\hline & [0.0153] & {$[0.0154]$} & {$[0.0154]$} & {$[0.0159]$} \\
\hline Observations & 447259 & 447259 & 443596 & 412607 \\
\hline$R^{2}$ & 0.033 & 0.044 & 0.043 & 0.046 \\
\hline Importer fixed effects & No & Yes & Yes & Yes \\
\hline Fuels excluded & No & No & Yes & Yes \\
\hline Baseline technology & non-classified & non-classified & non-classified & low \\
\hline
\end{tabular}

Standard errors in brackets; ${ }^{*} p<.10,{ }^{* \star} p<.05,{ }^{* \star *} p<.01$; Fuels refer to HS 2-digit product 27: Mineral fuels, mineral oils and products of their distillation; bituminous substances; mineral waxes. 


\section{Robustness}

In the robustness section we challenge our findings ${ }^{10}$ by (i) using unconstrained import data,

(ii) performing separate regressions for the pre- and the post-crisis period, and finally by (iii) running separate regressions for four income groups as classified by the World Bank.

\subsection{THRESHOLDS FOR IMPORT DATA}

Our benchmark specification does not consider observations for products that never exceeded an import value of 10,000 USD for an importer during 1995 and 2014. The reason is that we do not want to bias our results with economically unimportant trade flows. However, using a too high threshold for imports might substantially decrease the number of importing countries in our sample for which elasticities can be computed, especially small island states. The threshold of 10,000 USD is arguably somewhat arbitrary. We therefore perform our robustness analysis for all reported import data without any restrictions, but bearing the risk of greater outlier values in mind.

Using all reported data without dropping observations with economically seemingly low import values, the number of initial fixed effects estimates would increase from 687,927 to 785,290 (i.e. by $14 \%$ ). After correcting estimates for endogeneity and a possible selection bias as well as dropping the tails of the distribution and positive elasticity estimates, the final number of import demand elasticity estimates increases from 548,625 to 603,433 estimates (i.e. by roughly $10 \%$ ). This means that not employing any restrictions on import values leaves us with a greater proportion of positive import demand elasticities to be excluded from our analysis.

$79 \%$ of the final estimates are found to be statistically significant at the $10 \%$ level, which is slightly less than in our preferred specification. Although median values are very similar, showing inelastic import demand elasticities of around -0.95 , mean values do differ. Using all import data the minimum value, i.e. for the most elastic product, is found at -99 . This means that a $1 \%$ increase in the price of the imported good leads to a $99 \%$ decrease in import quantities. Excluding import values below 10,000 USD reduces this minimum value to -25 . The scaling up of import demand elasticities when including smaller import values is what we expect, recalling from equation (16) that $\hat{\varepsilon}_{n n i}=\widehat{a_{n n}} / \overline{s_{n l}}+\overline{s_{n l}}-1$ with $a_{n n}^{t}$ being a product-specific term that is equal for all countries.

\subsection{THE DIFFERENCE BETWEEN PRE- AND POST-CRISIS ESTIMATES}

Our investigation encompasses data from 1995 to 2014. This period notably includes the world financial and economic crisis, which might have had a significant impact on the elasticity of demand for imported products. Therefore, we split our sample into a pre-crisis period covering the years 1995-2007 and a post-crisis period 2009-2014 and estimated elasticities separately for both time spans.

10 Based on FE estimation before correction for endogeneity and self-selection. 
Comparing results for the pre-crisis with the post-crisis period, we still find a rather inelastic mean elasticity of -0.95 for both subsamples. However, while the mean elasticity for the pre-crisis period is found at around -1.7 , the post-2008 period shows a higher mean elasticity of -2.4 . We find that the discrepancy in mean elasticities between the pre- and post-crisis period is particularly strong for intermediate products. Looking at simple average binding elasticities, we find demand for imports of intermediate goods to be $13 \%$ more elastic in the post-2008 period compared to the period in the run-up to the financial crisis. Demand for goods attributable to GFCF also appears slightly more elastic for the post-crisis period, by roughly $1.2 \%$, but remains rather inelastic with an average elasticity around -0.87 . Only the demand for final consumption goods shows a $1.7 \%$ lower average elasticity after the crisis.

\subsection{DIFFERENTIATION BY THE LEVEL OF ECONOMIC DEVELOPMENT}

The GDP function approach proposed by Kee et al. (2008) assumes that the GDP function is common across all countries up to a country-specific term. This implies that $\hat{a}_{n n}$ in equation (11) which captures the change in the share of good $n$ in GDP resulting from a price increase of good $n$ by $1 \%$ is equal across countries.

Figure 6 / Binding elasticities over income: estimates per income group
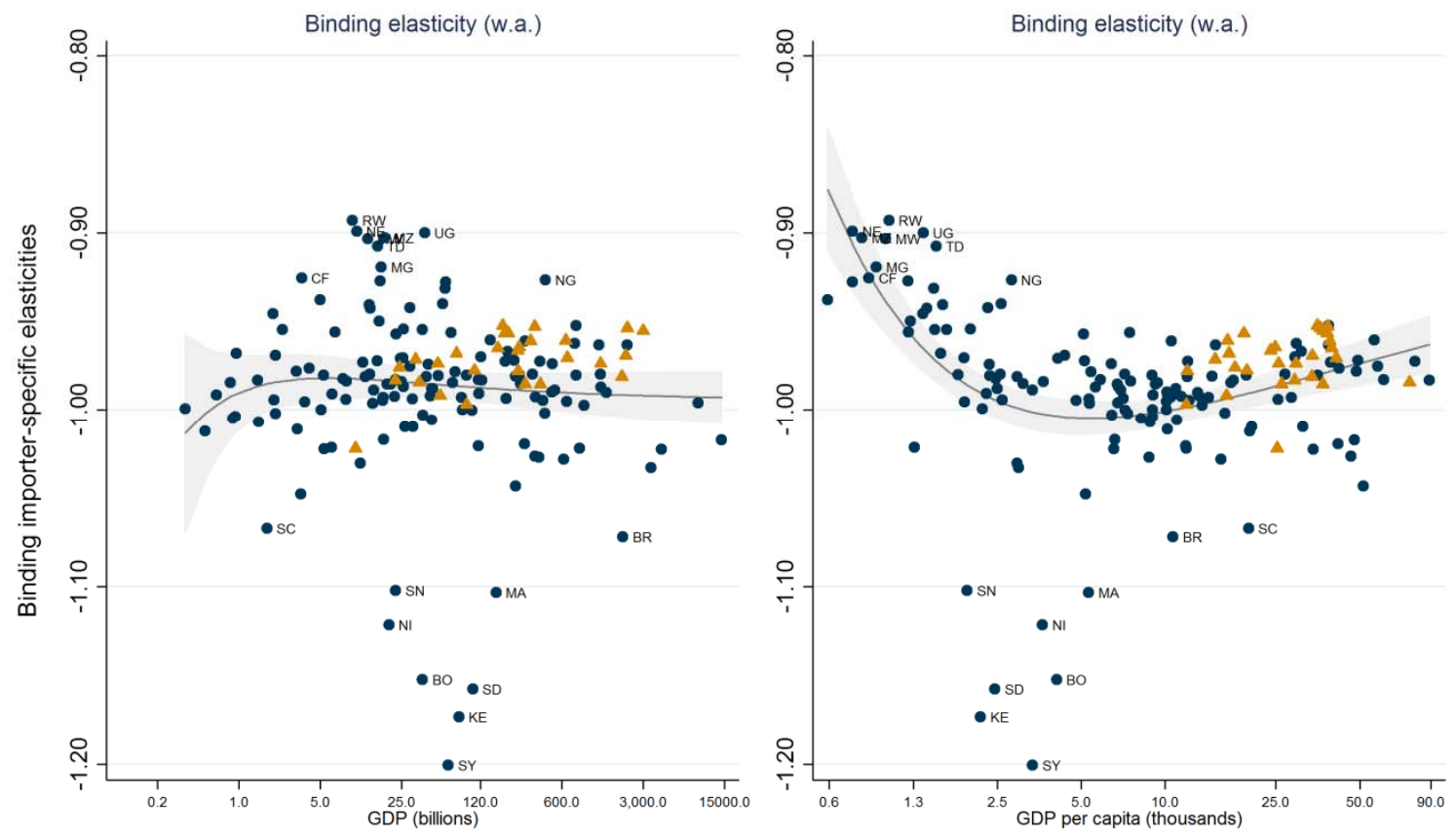

Note: w.a. refers to the import-weighted average per country: $\sum_{h}\left(\hat{\varepsilon}_{n n i} x\right.$ Imports $_{i h} /$ Imports $\left._{i}\right)$. Binding elasticities refer to estimates significantly different from zero at the 10\% level. GDP p.c. refers to the average expenditure-side real GDP per capita measured at chained PPPs in thousand 2011 USD for the period 1995-2014. EU Member States highlighted as orange triangles.

The variability of the product composition of imports might however differ by countries. Specifically, we expect it to vary by the level of economic development of the importing country. We therefore rerun our estimations for four different income groups as classified in the World Bank list of economies (version 
Wiiw Working Paper 132

July 2015): (i) low-income, (ii) lower-middle-income, (iii) upper-middle-income and (iv) high-income groups. ${ }^{11}$ The results are shown in Figure 6.

This specification improves the fit for binding elasticity estimates plotted against GDP per capita in the right panel. In particular, the poorest countries in our sample seem to be most price-inelastic with respect to imports, with middle-income countries being centred on an elasticity of -1 and high-income countries again showing less elastic demand. Middle-income countries showing elasticities greater than -1.1 comprise Syria, Kenia, Sudan, Bolivia, Nicaragua, Senegal, and Morocco.

11 For the determination of income group thresholds and data on their evolution over time please consult: https://datahelpdesk.worldbank.org/knowledgebase/articles/378833-how-are-the-income-group-thresholds-determined 


\section{Conclusion}

In this paper, we present import demand elasticities estimated for 167 countries and 5124 products at the six-digit level of the Harmonised System revision 1996. Following the semiflexible translog GDP function approach proposed by Kee et al. (2008), we estimate unilateral import demand elasticities for the period 1996-2014. Estimates by Kee et al. (2008) cover 117 countries for about 4900 products at the HS 6-digit level. This paper constitutes an update of their work for the more recent period 1996-2014. Improved data availability and the inclusion of products not considered in HS revision 1988 allow us to estimate about twice as many import demand elasticities. The presented results are differentiated by country and product characteristics.

Looking at the geographical distribution of import demand elasticities, simple averages indicate that South Asia and North America are associated with the most elastic import demand. Countries exhibiting the highest average elasticities belong to the economically most important countries in their respective regions, while countries with the lowest import demand elasticities are small island states with the exception of landlocked and poverty-ridden Chad. Import-weighted results suggest that especially countries rich in natural resources - particularly fossil fuels - are facing an inelastic import demand, with the agri-food sector for these states being more price-responsive than the manufacturing sector. Europe, too, is characterised by a rather inelastic import demand, particularly for Eastern European countries and the Iberian Peninsula.

Both the European Union and the United States show the highest elasticities for live animals, animal and vegetable fats and mineral products, but with the United States facing an import demand about twice as elastic. Inelastic demand is found for luxury goods such as pearls or works of art, machinery and electrical equipment, arms and ammunition and in the case of the EU but not the US for vehicles and aircrafts. Applying the product classification according to industries used in the WIOD, the energy sectors again feature as the most elastic, while imports of electrical equipment and machinery are found to be price-inelastic. Distinguishing between the use of products, it is evident that intermediate goods face the highest elasticities, which appears particularly noteworthy in the context of an increasing importance of global value chains and production fragmentation, the global trade slowdown since 2011 and ongoing negotiations of mega-regional trade deals.

Our preferred specification does not include importer-product observations where imports of a particular product to one specific importer never exceeded 10,000 USD between 1995 and 2014. Using all data provided by UN Comtrade without any import threshold has no impact on the median of the distribution but results in higher mean elasticities with the minimum elasticity shifting from about -25 to -99 . Splitting the period 1995-2014 into a pre- and post-crisis period indicates that after 2008 import demand became more elastic, particularly for intermediate goods. A final specification suggests that allowing the effect of prices on the product composition of GDP to vary by the economic development of countries along the income group classification of the World Bank, suggests that import demand elasticity is U-shaped. The poorest countries seem to be the least price-responsive with respect to imports, while the majority of middle-income countries is centred around unitary elasticity, with richer countries again being less sensitive to price changes. 


\section{References}

Altinay, G. (2007), 'Short-run and long-run elasticities of import demand for crude oil in Turkey', Energy Policy, Vol. 35, pp. 5829-5835.

Beghin, J., A.-C. Disdier and S. Marette (2015), 'Trade Restrictiveness Indices in Presence of Externalities: An Application to Non-Tariff Measures', Canadian Journal of Economics, Vol. 48, No. 4, November, pp. 1513-1536.

Bratt, M. (2014), 'Estimating the bilateral impact of non-tariff measures (NTMs)', Working Paper WPS 14-01-1, Université de Genève.

Broda, C. and D.E. Weinstein (2006), 'Globalization and the gains from variety', The Quarterly Journal of Economics, Vol. 121, No. 2, pp. 541-585.

Caves, D.W., L.R. Christensen and W.E. Diewert (1982), 'Multilateral comparisons of output, input, and productivity using superlative index numbers', The Economic Journal, Vol. 92, No. 365, March, pp. 73-86.

Diewert, W.E. and T.J. Wales (1988), 'A normalized quadratic semiflexible functional form', Journal of Econometrics, Vol. 37, No. 3, pp. 327-342.

Evenett, S.J. and J. Fritz (2016), Global Trade Plateaus. The $19^{\text {th }}$ Global Trade Alert Report, CEPR Press, London.

Feenstra, R.C. and J. Romalis (2014), 'International Prices and Endogenous Quality', The Quarterly Journal of Economics, Vol. 129, No. 2, May, pp. 477-527.

Feenstra, R.C., R. Inklaar and M.P. Timmer (2015), 'The Next Generation of the Penn World Table', American Economic Review, Vol. 105, No. 10, pp. 3150-3182.

Hatzichronoglou, T. (1997), 'Revision of the High-Technology Sector and Product Classification', OECD Science, Technology and Industry Working Papers, No. 1997/02.

Hausmann, R., C.A. Hidalgo, S. Bustos, M. Coscia, S. Chung, J. Jimenez, A. Simoes and M. Yildirim. (2011), The Atlas of Economic Complexity, Puritan Press, Cambridge MA.

Kee, H.L., A. Nicita and M. Olarreaga (2008), 'Import demand elasticities and trade distortions', The Review of Economics and Statistics, Vol. 90, No. 4, pp. 666-682.

Kee, H.L., A. Nicita and M. Olarreaga (2009), 'Estimating Trade Restrictiveness Indices', The Economic Journal, Vol. 119, pp. 172-199.

Kohli, U. (1991), Technology, Duality, and Foreign Trade: The GNP Function Approach to Modeling Imports and Exports, The University of Michigan Press, Ann Arbor.

Maoz, Z. (2009), 'The effects of strategic and economic interdependence on international conflict across levels of analysis', American Journal of Political Science, Vol. 53, No. 1, pp. 223-240.

Nizovtsev, D. and A. Skiba (2016), 'Import Demand Elasticity and Exporter Response to Anti-Dumping Duties', The International Trade Journal, Vol. 30, No. 2, pp. 83-114.

Panagariya, A., S. Shah and D. Mishra (2001), 'Demand elasticities in international trade: are they really low?', Journal of Development Economics, Vol. 64, pp. 313-342.

Peterson, T.M. and C.G. Thies (2014), 'The Demand for Protectionism: Democracy, Import Elasticity, and Trade Barriers', International Interactions, Vol. 40, pp. 103-126. 
Schaffer, M.E. (2010), xtivreg2: Stata module to perform extended IV/2SLS, GMM and AC/HAC, LIML and k-class regression for panel data models, http://ideas.repec.org/c/boc/bocode/s456501.html.

Soderbery, A. (2015), 'Estimating import supply and demand elasticities: Analysis and implications', Journal of International Economics, Vol. 96, pp. 1-17.

Timmer, M.P., E. Dietzenbacher, B. Los, R. Stehrer and G.J. de Vries (2015), 'An Illustrated User Guide to the World Input-Output Database: the Case of Global Automotive Production', Review of International Economics, Vol. 23, No. 3, pp. 575-605. 
wiiw Working Paper 132

\section{Appendix}

\section{Appendix 1 / Regional classification of countries}

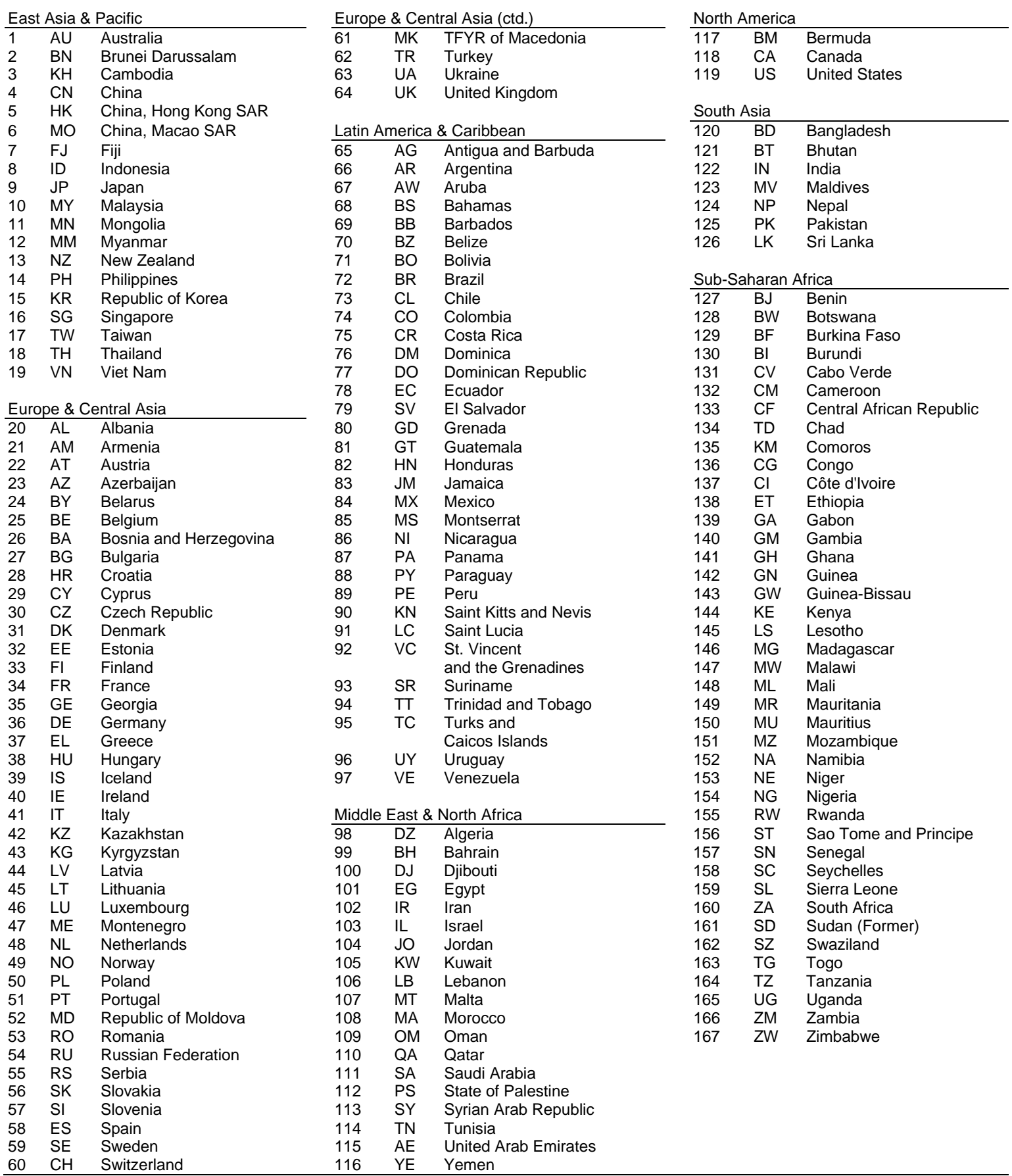

Note: World Bank list of economies (July 2015), Montserrat not classified by the World Bank. Information on West Bank and Gaza used for Palestine. 
Appendix 2 I Income classification of countries

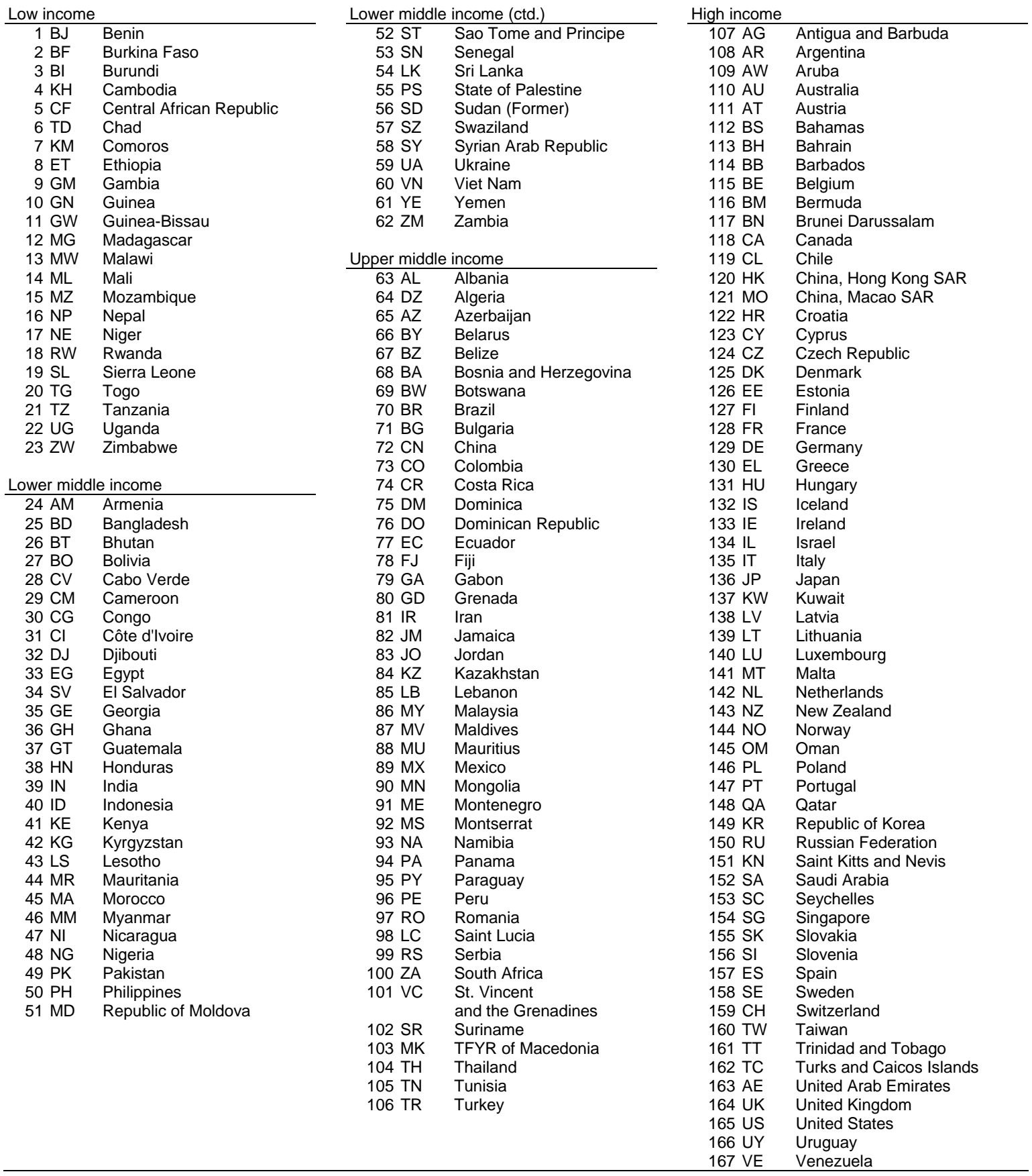

Note: World Bank list of economies (July 2015), Montserrat classified according to information provided by the United Nations. Information on West Bank and Gaza used for Palestine. 


\section{WIIW WORKING PAPERS PUBLISHED SINCE 2013}

For current updates and summaries see also wiiw's website at www.wiiw.ac.at

No. 132 Mahdi Ghodsi, Julia Grübler and Robert Stehrer: Import Demand Elasticities Revisited, November 2016

No. 131 Leon Podkaminer: Has Trade Been Driving Global Economic Growth?, October 2016

No. 130 Philipp Heimberger: Did Fiscal Consolidation Cause the Double-Dip Recession in the Euro Area?, October 2016

No. 129 Julia Grübler, Mahdi Ghodsi and Robert Stehrer: Estimating Importer-Specific Ad Valorem Equivalents of NonTariff Measures, September 2016

No. 128 Sebastian Leitner and Robert Stehrer: Development of Public Spending Structures in the EU Member States: Social Investment and its Impact on Social Outcomes, August 2016

No. 127 Roman Stöllinger: Structural Change and Global Value Chains in the EU, July 2016

No. 126 Jakob Kapeller, Michael Landesmann, Franz X. Mohr and Bernhard Schütz: Government Policies and Financial Crises: Mitigation, Postponement or Prevention?, May 2016

No. 125 Sandra M. Leitner and Robert Stehrer: The Role of Financial Constraints for Different Innovation Strategies: Evidence for CESEE and FSU Countries, April 2016

No. 124 Sandra M. Leitner: Choosing the Right Partner: R\&D Cooperations and Innovation Success, February 2016

No. 123 Michael Landesmann, Sandra M. Leitner and Robert Stehrer: Changing Patterns in M\&E-Investment-Based Innovation Strategies in CESEE and FSU Countries: From Financial Normalcy to the Global Financial Crisis, February 2016

No. 122 Sebastian Leitner: Drivers of Wealth Inequality in Euro-Area Countries. The Effect of Inheritance and Gifts on Household Gross and Net Wealth Distribution Analysed by Applying the Shapley Value Approach to Decomposition, January 2016

No. 121 Roman Stöllinger: Agglomeration and FDI: Bringing International Production Linkages into the Picture, December 2015

No. 120 Michael Landesmann and Sandra M. Leitner: Intra-EU Mobility and Push and Pull Factors in EU Labour Markets: Estimating a Panel VAR Model, August 2015

No. 119 Michael Landesmann and Sandra M. Leitner: Labour Mobility of Migrants and Natives in the European Union: An Empirical Test of the 'Greasing of the Wheels' Effect' of Migrants, August 2015

No. 118 Johannes Pöschl and Katarina Valkova: Welfare State Regimes and Social Determinants of Health in Europe, July 2015

No. 117 Mahdi Ghodsi: Distinguishing Between Genuine and Non-Genuine Reasons for Imposing TBTs; A Proposal Based on Cost Benefit Analysis, July 2015

No. 116 Mahdi Ghodsi: Role of Specific Trade Concerns on TBT in the Import of Products to EU, USA, and China, June 2015

No. 115 Mahdi Ghodsi: Determinants of Specific Trade Concerns Raised on Technical Barriers to Trade, June 2015

No. 114 Sandra M. Leitner and Robert Stehrer: What Determines SMEs' Funding Obstacles to Bank Loans and Trade Credits? A Comparative Analysis of EU-15 and NMS-13 Countries, May 2015

No. 113 Sebastian Leitner: Effects of Income Inequality on Population Health and Social Outcomes at the Regional Level in the EU - Differences and Similarities between CEE and Non-CEE EU Regions, May 2015

No. 112 Arne J. Nagengast and Robert Stehrer: The Great Collapse in Value Added Trade, April 2015

No. 111 Michael Landesmann, Sandra Leitner and Isilda Mara: Should I Stay, Should I Go Back or Should I Move Further? Contrasting Answers under Diverse Migration Regimes, January 2015

No. 110 Robert Stehrer: Does the Home Bias Explain Missing Trade in Factors? December 2014

No. 109 Sebastian Leitner and Robert Stehrer: Labour Market Transitions of Young People during the Economic Crisis, November 2014

No. 108 Neil Foster-McGregor, Johannes Pöschl and Robert Stehrer: Capacities and Absorptive Barriers for International R\&D Spillovers through Intermediate Inputs, October 2014

No. 107 Arne J. Nagengast and Robert Stehrer: Collateral Imbalances in Intra-European Trade? Accounting for the Differences between Gross and Value Added Trade Balances, July 2014

No. 106 Roman Stöllinger and Mario Holzner: State Aid and Export Competitiveness in the EU, December 2013

No. 105 Neil Foster-McGregor, Anders Isaksson and Florian Kaulich: Importing, Productivity and Absorptive Capacity in Sub-Saharan African Manufacturing Firms, November 2013

No. 104 Isilda Mara and Michael Landesmann: The Steadiness of Migration Plans and Expected Length of Stay - Based on a Recent Survey of Romanian Migrants in Italy, September 2013

No. 103 Isilda Mara and Michael Landesmann: Do I Stay because I am Happy or am I Happy because I Stay? Life Satisfaction in Migration, and the Decision to Stay Permanently, Return and Out-migrate, August 2013

No. 102 Rod Falvey and Neil Foster-McGregor: On the Trade and Price Effects of Preferential Trade Agreements, May 2013 

IMPRESSUM

Herausgeber, Verleger, Eigentümer und Hersteller:

Verein "Wiener Institut für Internationale Wirtschaftsvergleiche“ (wiiw),

Wien 6, Rahlgasse 3

ZVR-Zahl: 329995655

Postanschrift: A 1060 Wien, Rahlgasse 3, Tel: [+431] 53366 10, Telefax: [+431] 533661050 Internet Homepage: www.wiiw.ac.at

Nachdruck nur auszugsweise und mit genauer Quellenangabe gestattet.

Offenlegung nach § 25 Mediengesetz: Medieninhaber (Verleger): Verein "Wiener Institut für Internationale Wirtschaftsvergleiche", A 1060 Wien, Rahlgasse 3. Vereinszweck: Analyse der wirtschaftlichen Entwicklung der zentral- und osteuropäischen Länder sowie anderer Transformationswirtschaften sowohl mittels empirischer als auch theoretischer Studien und ihre Veröffentlichung; Erbringung von Beratungsleistungen für Regierungs- und Verwaltungsstellen, Firmen und Institutionen. 
wiiw

wiiw.ac.at 